\title{
ALGEBRAIC PARTICULAR INTEGRALS, INTEGRABILITY AND THE PROBLEM OF THE CENTER
}

\author{
DANA SCHLOMIUK
}

\begin{abstract}
In this work we clarify the global geometrical phenomena corresponding to the notion of center for plane quadratic vector fields. We first show the key role played by the algebraic particular integrals of degrees less than or equal to three in the theory of the center: these curves control the changes in the systems as parameters vary. The bifurcation diagram used to prove this result is realized in the natural topological space for the situation considered, namely the real four-dimensional projective space. Next, we consider the known four algebraic conditions for the center for quadratic vector fields. One of them says that the system is Hamiltonian, a condition which has a clear geometric meaning. We determine the geometric meaning of the remaining other three algebraic conditions (I), (II), (III). We show that a quadratic system with a weak focus $F$, possessing algebraic particular integrals not passing through $F$ of the following types, satisfies in some coordinate axes the condition (I), (II) or (III) respectively and hence has a center at $F$ : either a parabola and an irreducible cubic particular integral having only one point at infinity, coinciding with the one of the parabola; or a straight line and an irreducible conic curve; or distinct straight lines (possibly with complex coefficients). We show that each one of these geometric properties is generic for systems satisfying the corresponding algebraic condition for the center. Another version of this result in terms of real algebraic curves is given. These results make clear the many facets of the problem of the center in the quadratic case, in particular the question of integrability and form a basis for analogous investigations for the general problem of the center for cubic systems.
\end{abstract}

\section{INTRODUCTION}

In [29], $\mathrm{H}$. Poincare defined the notion of a center for a real vector field on the plane (i.e., an isolated singularity surrounded by closed integral curves) and he showed (cf. [30]) that a necessary and sufficient condition for a polynomial vector field with a singular point with pure imaginary eigenvalues, to have a center at this point is the annihilation of an infinite number of polynomials in the coefficients of the vector field. The problem of explicitly finding a finite basis for these algebraic conditions (the problem of the center), was solved in the case of quadratic vector fields by the successive contributions of $\mathrm{H}$. Dulac,

Received by the editors November 15, 1990 and, in revised form, February 12, 1991 and May 17, 1991.

1991 Mathematics Subject Classification. Primary 58F14, 58F22, 34C05.

Key words and phrases. Algebraic particular integral, constant of motion, center, bifurcation, polynomial system, quadratic system.

Partially supported by NSERC and by Quebec Education Ministry. 
W. Kapteyn, N. Bautin, N. A. Sakharnikov, L. N. Belyustina, and K. S. Sibirsky. Each quadratic system with a center admits a nonconstant analytic first integral defined on $\mathbb{R}^{2}$ or on the complement of an algebraic curve [37]. Thus, each one of the finite algebraic conditions for the center is a criterion for integrability: to prove integrability on a dense open set of the plane, it suffices to show that a system verifies one of the finite number of algebraic conditions for a center. For this reason the conditions for the center are sometimes called the integrability conditions.

The problem of the center plays an important role in the second part of Hilbert's 16th problem (cf. [15]), which asks for the maximum number of limit cycles which a vector field given by polynomials of degree $d$ could have. One way to produce limit cycles is by perturbing a system which has a continuous family of closed orbits, in such a way as to "create" limit cycles in the perturbation from some of the closed orbits in the original system (cf. [31, 14]). Each system with a center has a continuous family of closed orbits and thus each system could "create" limit cycles in perturbations. An understanding of these systems, gives us an idea of how vast the second part of Hilbert's 16th problem really is. This problem is still unsolved even for the quadratic case. An attempted solution for this problem for the quadratic case, published by Petrovski and Landis in the 1950s (cf. [28]), contained errors and the authors acknowledged a gap in [21]. Recently another claim for a solution to this problem was made in [6] but again the "proof" does not meet the necessary standards of rigour. Indeed, Il'yashenko [16] and Shi Songling [38] reported that they found errors in [6] or in material on which [6] is based.

In this work we focus our attention on the global geometry of the real plane quadratic vector fields with a center. The problem we consider in this work is that of finding a geometrical meaning for each one of the finite number of the algebraic conditions for the center, for plane quadratic vector fields. It is this question which in the end ties together the key elements connected with the problem of the center, i.e., the algebraic conditions for the center, the question of integrability of the systems and the invariant algebraic curves which they possess.

The space $\mathbf{Q}$ of all plane quadratic vector fields is an open set of a 12dimensional vector space formed by the coefficients of the quadratic polynomials. In $\mathbf{Q}$ lies the subspace $\mathbf{C}$ of all the quadratic systems with a center. The groups of affine coordinate transformations of the plane and of positive rescaling of time, act on $\mathbf{Q}$. The orbit space $\widetilde{\mathbf{Q}}$ of the group action is fivedimensional and in $\widetilde{\mathbf{Q}}$ lies the orbit space $\widetilde{\mathbf{C}}$ of the space $\mathbf{C}$. The singular points of quadratic systems, which have at least one zero eigenvalue cannot be centers (cf. $[4,43,17])$. So we consider quadratic systems with a singular point with nonzero eigenvalues. Such a singular point could be a center only if the eigenvalues are pure imaginary in which case we call such a singular point a weak focus. We denote by $\widetilde{\mathfrak{F}}$ the orbit space, under the group action, of the space $\mathfrak{F}$ of all quadratic systems possessing a weak focus $F$. When quadratic systems with a weak focus $F$ are placed in a certain canonical form, the form of Kapteyn (cf. $\S 3$ ), with $F$ placed at the origin, they depend on five parameters which we shall denote by $a, b, d, A, C$ and which appear as coefficients of the quadratic polynomials defining these systems, one of them being nonzero for 
a nonlinear system. Since homotheties of the two coordinate axes do not change the phase portraits of these systems, the parameter space is actually the fourdimensional projective space, real or complex according to whether we take real or complex coefficients and $a, b, d, A, C$ are homogeneous coordinates of this space. In this work we use the conditions for the center for quadratic systems given by Kapteyn and Bautin (cf. [3]). The set of all quadratic systems with a center is an algebraic set in the parameter space, defined by a set of polynomial equations with rational coefficients, in $a, b, d, A, C$. The four conditions for the center of Kapteyn-Bautin correspond to the four irreducible components of this algebraic set. One of these four conditions says that the system is Hamiltonian and we denote this condition by $(\mathrm{H})$ and the complex projective algebraic variety it defines by $\mathbf{V}_{\mathbf{H}} \cdot \mathbf{V}_{\mathbf{H}}$ is two-dimensional and it is a plane in the parameter space. Since the case of the Hamiltonian systems it is clear that algebraic curves control the behaviour of the systems (indeed the integral curves lie on cubic curves, the level curves of the cubic Hamiltonian of the systems), we leave aside this case and we concentrate our attention on the remaining other three conditions for the center, trying to capture their geometrical meaning. The remaining three conditions for the center determine sets which are algebraic varieties of dimensions three, two, and one, in the parameter space. We denote these conditions respectively by (III), (II), and (I) (cf. §3) and the algebraic varieties which they define in the complex projective space by $\mathbf{V}_{\text {III }}, \mathbf{V}_{\text {II }}$, and $\mathbf{V}_{\text {I }}$ respectively. $\mathbf{V}_{\text {III }}$ is a hyperplane in the parameter space, $V_{I I}$ is a plane and $V_{I}$ is an irreducible plane conic curve. Since we are concerned with systems possessing a center, notion which as defined by Poincaré refers to real systems, we consider the real part of $\mathbf{V}_{i}$, which we denote by $\mathbf{C}_{i}$, $i=\mathrm{I}, \mathrm{II}, \mathrm{III}, \mathrm{H}$. We may identify the points of $\mathbf{C}_{i}$ with the quadratic systems to which they correspond. A look at the polynomial equations defining one of the conditions (III), (II), or (I) does not give us any clue about the geometrical nature of the quadratic systems satisfying them. We set out to determine this geometrical nature. For systems in $\mathfrak{F}$ we consider three geometric properties $\mathbf{P}_{\text {I }}, \mathbf{P}_{\text {II }}, \mathbf{P}_{\text {III }}$, each saying that the system has two particular integrals, not passing through $F$ and which are respectively of the following types: For $\mathbf{P}_{I}$ the particular integrals are a parabola and an irreducible cubic, having only one point at infinity, coinciding with the one of the parabola; For $\mathbf{P}_{\mathrm{II}}$ they are a straight line and an irreducible conic curve; For $\mathbf{P}_{\text {III }}$ the particular integrals are two distinct straight lines, possibly with complex coefficients. Let us denote by $\mathfrak{F}_{i}$, for $i=\mathrm{III}, \mathrm{II}, \mathrm{I}$, the set of systems in $\mathfrak{F}$ which satisfies the property $\mathbf{P}_{i}$. The results of this paper concern the relationship between the sets $\mathbf{C}_{i}$ 's defined by the conditions for the center and the sets $\mathfrak{F}_{i}$ 's defined by the geometrical properties $\mathbf{P}_{i}$ 's. We show that a system in $\mathfrak{F}_{i}$ satisfies in some coordinate system the condition $i=\mathrm{I}$, II, III, i.e., the orbit of the system, under the group action, contains an element in $\mathbf{C}_{i}$. Furthermore, we show the generic nature of these properties $\mathbf{P}_{i}$. For a given topological space $X$, a property $P$ is generic if the subset of $X$ which it defines contains an intersection of open dense subsets of $X$ (cf. [24] or [1]). Then the three geometrical properties $\mathbf{P}_{I}, \mathbf{P}_{\text {II }}, \mathbf{P}_{\text {III }}$ are generic in the sets $\mathbf{C}_{I I I}, \mathbf{C}_{I I}, \mathbf{C}_{I}$ respectively. With the possible exception of the bifurcation points, systems belonging to $\mathbf{C}_{i}$ satisfy $\mathbf{P}_{i}$.

This work establishes a clear link between the theory of polynomial vector fields and algebraic curves, link which appears in the theory of the center for 
quadratic systems. Such links were suggested by the way Hilbert stated his 16 th problem by splitting it into two parts: the first one about the topology of algebraic curves in the real projective plane and the second part about the maximum number of limit cycles of vector fields defined by real polynomials of degree $d$ (cf. [15]). As we see in this work, algebraic curves as well as higher dimensional algebraic varieties appear naturally in the theory of polynomial vector fields, establishing connections between this theory and algebraic geometry.

Secondly, this work makes clear the relation between center and integrability of the system on a dense open set, in the quadratic case. Indeed, in [7] Darboux showed how the first integrals of algebraic first order differential equations possessing enough algebraic integrals are constructed. Placed in the perspective of Darboux's work, this work explains in the generic case, why the quadratic systems with a center are integrable.

Finally, this work provides us with a background for work on the problem of the center for general cubic systems, problem which is still open. The cubic systems with a center at a weak focus determine an algebraic set in the parameter space of the coefficients of the systems. Attempts, using the computer, to determine a finite basis for the ideal $\mathfrak{I}$ defining this algebraic set have ended up in failure, the calculations leading to enormous expressions after the first few elements of this basis. Since the irreducible components of the algebraic set of quadratic systems with centers are linked here with certain types of algebraic particular integrals of the systems involved, it is natural to use algebraic particular integrals in exploring conditions for the center in the case of cubic systems, bypassing (in the first instance) the problem of calculating the very lengthy polynomials in the finite basis of the ideal $\mathfrak{I}$.

The geometrical results we obtained were first reached with the help of the computer algebra, using MACSYMA and a VAX/780 computer. The calculations were later simplified and we bring them here in ordinary proof form. In fact the proofs we give here are completely elementary.

The work is organized as follows: In $\S 2$ we review briefly the paper of Dulac [9] which is the first work to appear in the literature on the center, after the memoirs of Poincaré [29], [30] and Lyapunov [27]. Both for historical reasons and because Dulac's article [9] brought into the picture all the essential ingredients of the problem of the center in the quadratic case, Dulac's paper deserved more attention than it has received in the past and we briefly discuss its results. In $\S 3$ we give the basic notions and results needed in this work; we state the theorem giving the four algebraic conditions for the center which we use, for quadratic vector fields. These are the Hamiltonian condition $(\mathrm{H})$ and the conditions which we denoted above by (III), (II), and (I). We also state the results on the integrability of these systems. The geometrical nature of these last three conditions was first seen by carefully analysing the bifurcation diagrams corresponding to these conditions. So in $\S 4$, we draw the bifurcation diagram corresponding to each one of the conditions for the center (III), (II), and (I). These diagrams are constructed in the real projective four-dimensional space. A two-dimensional subspace of the four-dimensional real projective space suffices for describing the bifurcation diagram of the quadratic systems with a center. In $\S 5$ we analyze the results obtained in $\S 4$ and we extract from them the key geometric features of the systems. These are the low degree algebraic particular integrals of these systems. We prove that all the bifurcation points in the pre- 
viously constructed diagrams are points in whose neighbourhood there occur changes, when we vary parameters, in the cubic curves, particular integrals of these systems. This shows the importance of these algebraic particular integrals in the theory of the center. We discuss the notion of algebraic particular integral and the calculation of such curves for polynomial vector fields in $\S 6$ where we illustrate the calculations by proving specific results for quadratic systems. In all these results we assume that the system possesses one algebraic particular integral of a specific type and from this assumption we deduce the conditions satisfied by the coefficients of the system. To obtain the conditions to have a center we need to assume the existence of two algebraic integrals of the specific types given by the properties $\mathbf{P}_{i}$ 's. This is done in $\S 7$ where we show that any real quadratic system with a weak focus, which satisfies one of the properties $\mathbf{P}_{i}$ also satisfies (in some coordinate system) the condition $i$, for $i=$ III, II, I and hence has a center. In view of the fact that with the possible exception of systems located at bifurcation points, all quadratic systems with a center are either Hamiltonian or they satisfy $\mathbf{P}_{i}$, for some $i=\mathbf{I}$, II, III, we obtain in this way a generic geometric characterization of the quadratic systems with a center, in terms of the algebraic particular integrals of the systems. This characterization clarifies the relationship between center and integrability and we discuss this relationship in $\S 8$ where we consider the results in the perspective of Darboux's work [7]. Darboux showed that knowledge of sufficiently many irreducible algebraic particular integrals gives us a first integral of the algebraic first order equations. In view of Darboux's work, the results in $\S 7$ also give us first integrals of quadratic systems with a center.

\section{THE PAPER OF DULAC ON THE INTEGRABILITY OF COMPLEX QUADRATIC EQUATIONS WITH A CENTER}

In [9], Dulac gives a complete list of first integrals for complex quadratic systems with a center. To obtain them, Dulac brings into the picture all the essential ingredients for a complete treatment of the problem of the center, for the quadratic case. One of these ingredients consists of the algebraic particular integrals of the equations. These integrals were first discussed by Darboux in [7]. Considering the long history of errors encountered in the literature on the center for quadratic systems (cf. [37]), it is clear that the work of Dulac [9] and the memoir of Darboux [7] did not receive the attention they deserved to have received. In this paragraph we discuss briefly Dulac's article [9] and we shall consider Darboux's work [7] in $\S 8$.

In [9], Dulac considers complex first order equations

$$
Y(x, y) d y+X(x, y) d x=0
$$

with $X$ and $Y$ holomorphic functions, which are zero for $x=y=0$ and he discusses the problem of finding general integrals for those equations (2.1) which possess a singularity of a kind which he calls a center. Dulac says that he uses the word center in a "larger sense" than the one used by Poincaré. Without making a formal definition, Dulac describes a center as follows: Consider an equation (2.1) which has a singular point with nonzero eigenvalues and let $\lambda$ be the quotient of the eigenvalues at this point. We may assume the singular point to be placed at the origin. After a linear change of variables, the differential 
equation may be assumed to be of the form

$$
(x+\cdots) d y+(-\lambda y+\cdots) d x=0 .
$$

Let $\lambda$ be a negative rational number $\lambda=-p / q, p, q>0, p, q \in \mathbb{Z}$. Dulac says: "If we try to put the general integral of the equation in the form $\phi(x, y)=$ $C, \phi$ verifies the equation

$$
(q x+\cdots) \frac{\partial \phi}{\partial x}-(p y+\cdots) \frac{\partial \phi}{\partial y}=0 .
$$

We could try to verify this equation with a holomorphic function $\phi$, zero for $x=y=0$." Considering the development of $\phi$ around the origin, the above equation yields an infinity of conditions which the coefficients of $\phi$ must satisfy, conditions obtained by annihilating the coefficients of the series in the left-hand side of the equation . Dulac says: "it is only if an infinity of conditions are satisfied, that (2.2) admits an integral of the indicated form. Only two integrals of the equation pass through the origin then. We are in the case of a center." In [9] he considers the case when $X, Y$ are quadratic polynomials and $\lambda=-1$. He shows that in this case for all values of the coefficients, the equation can be integrated "in finite terms". To show this implication, Dulac derives the first ones of the infinite number of conditions obtained from (2.3) when (2.1) is with $X, Y$ quadratic polynomials and $\lambda=-1$. He then uses these conditions to integrate the equations "in finite terms" either directly, or by making a change of variable or he uses the method of the algebraic particular solutions introduced by Darboux [7]. A discussion according to cases follows, the equations are classified according to seven normal forms which he then integrates and a complete list of integrals is obtained.

All the essential ingredients for a complete treatment of the problem of the center in the quadratic case appear in [9]. But in Dulac's treatment, these aspects are mixed at almost every stage of the work and in the end, a clear picture of the problem of the center does not emerge. For instance one does not come out with a finite number of necessary and sufficient conditions for the center for the quadratic case. The algebraic integrals appear in some instances in order to perform the integration but their fundamental role and the relationship connecting the various elements used in the work is not clear. The aspect investigated in full by Dulac, the integrability "in finite terms" of the systems comes almost as a surprise for Dulac. In fact, as we shall later see, it is the geometry of the algebraic particular integrals which establishes clearly the relationships between the algebraic conditions for the center and the integrability of the systems with a center.

\section{The Algebraic CONDITIONS FOR A CENTER AND}

THE INTEGRABILITY OF THE QUADRATIC SYSTEMS WITH A CENTER

Definition 3.1. A singular point $F$ of a system

$$
d x / d t=f(x, y), \quad d y / d t=g(x, y)
$$

is a weak focus if the eigenvalues of the linearization at $F$ are pure imaginary.

The weak foci of quadratic systems are either centers or they are classified according to their order. To define the order we shall use of the following lemma [39]. 
Lemma 3.1. For a polynomial system

$$
\begin{aligned}
& \frac{d x}{d t}=-y+P_{2}(x, y)+\cdots+P_{n}(x, y), \\
& \frac{d y}{d t}=x+Q_{2}(x, y)+\cdots+Q_{n}(x, y)
\end{aligned}
$$

there exists a formal power series $F(x, y)$ such that

$$
\frac{d F}{d t}=V_{1}\left(x^{2}+y^{2}\right)^{2}+V_{2}\left(x^{2}+y^{2}\right)^{3}+\cdots+V_{n}\left(x^{2}+y^{2}\right)^{n+1}+\cdots
$$

where

$$
\frac{d F}{d t}=\frac{\partial F}{\partial x} P(x, y)+\frac{\partial F}{\partial y} Q(x, y)
$$

and $P, Q$ are respectively the right-hand sides of the equations (3.2).

An algebraic proof of this lemma, is given in [32]. The above lemma, stated exactly as in [32], is given without specifying where the coefficients of the $P_{i}$ 's, $Q_{i}$ 's and of the series $F(x, y)$ are to be taken. If the system (3.1) is real, this lemma is implicit in Poincarés work on the center (cf. p. 96 of [30]), where an analytic proof for it can be found. Due to its purely algebraic character, the content of this lemma also works if we consider the coefficients of the $P_{i}$ 's, $Q_{i}$ 's as being indeterminates and $F$ as a power series in $x, y$ with coefficients in the ring of polynomials with rational coefficients in these indeterminates.

For a system (3.2), the $V_{i}$ 's are polynomials in the coefficients of the system, with rational coefficients. These $V_{i}$ 's are not uniquely determined. For each $i$ there is an infinite number of possibilities for a $V_{i}$. But all such $V_{i}$ 's are in the same coset modulo the ideal generated by $V_{1}, V_{2}, \ldots, V_{i-1}$ in the ring of polynomials with rational coefficients in the coefficients of the system (3.2). In [40] this coset is called the $i$ th Poincaré-Lyapunov constant.

By Hilbert's basis theorem, the ideal of all $V_{i}$ 's in the ring of polynomials with rational coefficients in the coefficients of the system (3.2), is finitely generated. It follows from the work of Bautin [3] that for quadratic systems this ideal is determined by the values of $V_{j}$ with $j \leq 3$. The first three coefficients $V_{j}$ for quadratic systems were computed for the first time by Bautin (cf. [3, 12]) but as Shi Songling observed (cf. [40]) there was an error of sign in the expression corresponding to $V_{3}$ given by Bautin and later calculations confirmed those of Shi Songling.

Definition 3.2. For a system (3.2), the origin is a weak focus of order $k$ with $k>0$, if $V_{i}=0$ for $i<k$ and $V_{k} \neq 0$. The origin is a degenerate weak focus if $V_{1}=0$.

It follows from the work of Poincaré [30] that a system (3.2) has a center at the origin if and only if $V_{i}=0$ for all $i$.

A quadratic system with a weak focus may be brought by affine changes of axes and time rescaling to the form

$$
\frac{d x}{d t}=-y-b x^{2}-C x y-d y^{2}, \quad \frac{d y}{d t}=x+a x^{2}+A x y+c y^{2} .
$$

This notation for the coefficients respects the notation encountered in the literature on the center. In this paper we investigate the global geometrical behaviour of the systems (3.S) having a center at the origin. To see this behaviour, 
we work with a coordinate system in which the $V_{i}$ 's and the conditions for the center take a very simple form, the coordinate system used by Kapteyn who observed that a system (3.S) can be brought by a rotation of axes to one of the same form but with $a+c=0$ [18]. We shall use this observation and in what follows we denote by $\left(3 . \mathrm{S}^{\prime}\right)$ a system $(3 . S)$ for which $c$ is $-a$. Bautin observed [3] that a consequence of Kapteyn's work [18, 19] is the following theorem:

Theorem 3.1 (Kapteyn, Bautin). A system (3.S') has a center at the origin if and only if one of the following four conditions is satisfied:

(III) $b+d=0$,

(II) $C=0=a$,

(I) $0=C+2 a=A+4 b+5 d=a^{2}+b d+2 d^{2}$,

(H) $A-2 b=C+2 a=0$.

The last condition is denoted by $(\mathrm{H})$ because a system $\left(3 . \mathrm{S}^{\prime}\right)$ is Hamiltonian if and only if the condition $(\mathrm{H})$ holds. In this case the system has the following constant of motion:

$$
-\left(x^{2}+y^{2}\right) / 2-b x^{2} y+a x y^{2}-d y^{3} / 3-a x^{3} / 3 .
$$

A system $\left(3 . \mathrm{S}^{\prime}\right)$ is determined by five parameters: $a, b, d, C$, and $A$. Since one of these parameters is nonzero for a nonlinear system, we may make this coefficient unity by homothety. Hence, the parameter space is in fact the real projective four-dimensional space in which conditions (III), (II), and (I) determine the sets $\mathbf{C}_{i}, i=$ III, II, I of dimensions 3, 2, and 1, which explains our notation for these conditions.

The following is a basis for the ideal generated by the $V_{i}, i=1,2, \ldots$ (cf. $[3,12])$, in the ring $\mathbb{Q}[a, b, d, A, C]$ :

$$
\begin{gathered}
B_{1}=(C+2 a)(b+d), \\
B_{2}=(b+d) a(A-2 b)(A+3 b+5 d), \\
B_{3}=(b+d)^{2} a(A-2 b)\left(a^{2}+2 d^{2}+b d\right) .
\end{gathered}
$$

We have:

$$
\begin{gathered}
V_{1}=k B_{1}, \\
V_{2} \equiv l B_{2} \quad \operatorname{modulo}\left(V_{1}\right), \\
V_{3} \equiv s B_{3} \quad \operatorname{modulo}\left(V_{1}, V_{2}\right)
\end{gathered}
$$

where $k, l$, and $s$ are positive rational constants.

In view of Bautin's work [3], for a system $\left(3 . S^{\prime}\right)$, the origin is a center if and only if for $i=1,2,3$ we have $V_{i}=0$, i.e., if and only if $B_{i}=0$ for $i=$ $1,2,3$. Clearly these conditions determine an algebraic set in the parameter space, whose irreducible components are the algebraic varieties determined by the conditions (III), (II), (I), (H).

Remark 3.1. There is no analogue of the Kapteyn-Bautin theorem for cubic systems. It is still unknown how many $V_{i}$ 's are needed in order to obtain a set of generators in this case. For the general cubic systems with a weak focus, after the first $V_{i}$ 's the calculations become massive and the formulas very lengthly when $i$ grows. 

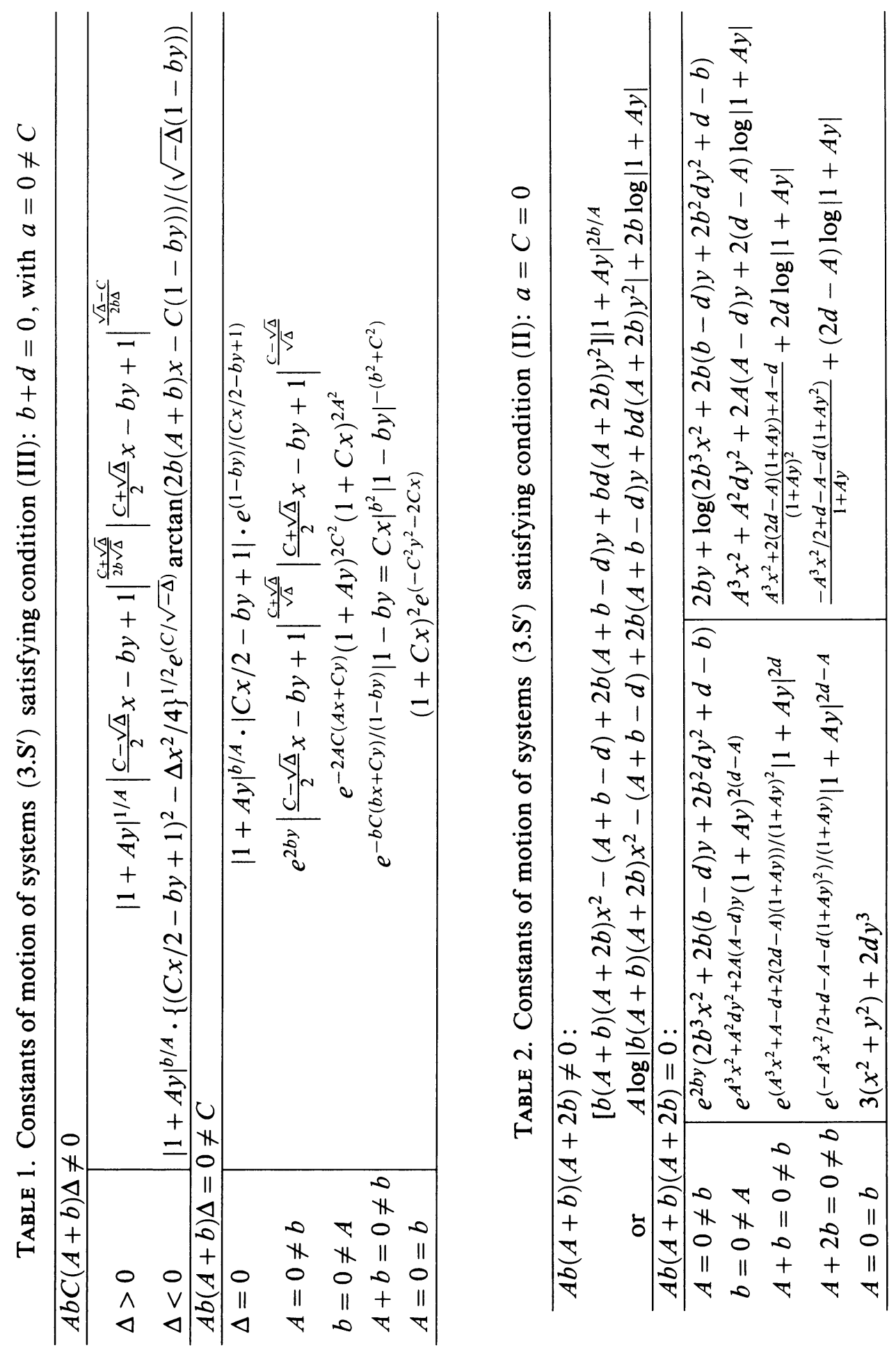
The paper of Dulac is concerned with complex systems but the ideas in this paper apply equally well to real or comples systems. The integrals for real quadratic systems were obtained by Lunkevich and Sibirsky [26].

Definition 3.3. A real (or complex) system (3.1) (or an equation $g(x, y) d x-$ $f(x, y) d y=0)$ is integrable on an open set $U$ of $\mathbb{R}^{2}$ (or of $\mathbb{C}^{2}$ ) if and only if there exists a nonconstant analytic function $F: U \rightarrow \mathbb{R}^{2}\left(\right.$ or $F: U \rightarrow \mathbb{C}^{2}$ ) which is constant on all solution curves $(x(t), y(t))$ in $U$, i.e., $F(x(t), y(t))=$ constant. (Other authors use $C^{1}$ functions which are not constant on any open subset of $U$, in place of our analytic function $F$.) Such an $F$ is called a first integral or a constant of motion on $U$.

Using this definition we have (cf. [37]):

Theorem 3.2. A real quadratic system (3.S) with a center is integrable on $\mathbb{R}^{2}$ or on the complement of an algebraic curve.

A complete list of constants of motions for quadratic systems with a center is formed by Tables 1 and 2 (which correspond to conditions (III) and (II)), the constant of motion given by the formula (3.4) for the Hamiltonian case and the rational function $(4.24)$ in $\S 4$, constant of motion corresponding to condition (I) for the case $a \neq 0$.

\section{BIFURCATION DIAGRAMS FOR QUADRATIC SYSTEMS WITH A CENTER}

$(\mathrm{H})$ is the only one of the four conditions for the center listed in Theorem 3.1, which has a clear geometric meaning: the integral curves of a system $\left(3 . S^{\prime}\right)$ satisfying this condition lie on cubic curves, the level curves of the cubic Hamiltonian of the system. Is there a geometrical meaning in each one of the other three conditions for the center? To see this, we draw bifurcation diagrams for conditions (III), (II), and (I) in Theorem 3.1 and then analyze these to see the geometrical properties which the phase portraits have in common.

Phase portraits of quadratic systems with a center were first drawn by Frommer [10]. Frommer's paper contains errors (we discussed these in [37]) and from the 32 phase portraits of the quadratic systems with a center he only drew 15 . Phase portraits of quadratic systems with a center appeared later in a sequence of papers [20, 22, 23, 25, 44]. We found errors in [25]. In [44] it is stated that the list in [20] contains a portrait which cannot be realized and the remaining ones do not form a complete list. The list in [44] turned out to be complete but a global dynamic picture does not emerge from this list since these phase portraits are not assembled in a bifurcation diagram. We obtained bifurcation diagrams corresponding to the systems with a center and those corresponding to the three conditions for the center (I), (II), (III) appeared in [32]. These diagrams lie in the real four-dimensional projective space.

To draw the phase portraits of the plane vector fields, we use the Poincaré compactification (cf. [29, 13]) of these on the sphere via the central projection. To be precise, the plane $Z=1$ in the space coordinates $X, Y, Z$ is projected on the sphere of radius one centered at the origin. We use the coordinates $x=X, y=Y$ in the plane $Z=1$. The central projection sends the point $(x, y)$ of this plane on two opposite points on the sphere. A vector field on the complement of the equator of the sphere is thus obtained and there exists an analytic vector field defined on the whole sphere (cf. [13]), which gives 
by a time rescaling the original polynomial vector field on the corresponding chart. We denote the coordinates in the associated affine local charts corresponding to points on the sphere belonging to $U_{X}=\{(X, Y, Z) \mid X \neq 0)$ and to $U_{Y}=\{(X, Y, Z) \mid Y \neq 0)$ respectively by $(u, z)$ and $(v, z)$. For points on the sphere belonging to $U_{Z}=\{(X, Y, Z) \mid Z \neq 0\}$ the local coordinates are $x, y$. The connecting maps between the charts are $(x, y)=(X, Y, 1) \rightarrow$ $(Y / X, 1 / X)=(u, z)$ and $(x, y)=(X, Y, 1) \rightarrow(X / Y, 1 / Y)=(v, z)$. Except for the orientation of the integral curves, the vector field behaves similarly on the north $(Z>0)$ and south $(Z<0)$ hemispheres. A compactification of our vector field is obtained from the restriction of the sphere field on the northern hemisphere completed by the equator. To view this field, we project this hemisphere vertically on the $(x, y)$ plane which is the plane $Z=1$ and we draw the phase portraits on the resulting disk where on the circumference we have the behaviour at infinity.

We consider now the parameter space where the bifurcation diagrams will be described. The space $\mathbf{Q}$ of all systems (3.1) with $f$ and $g$ quadratic polynomials is an open set of a 12-dimensional vector space formed by the coefficients of $f$ and $g$. The groups of affine coordinate transformations of the plane and of positive rescaling of time, act on $\mathbf{Q}$. The orbit space $\widetilde{\mathbf{Q}}$ of the group action is five-dimensional and in $\widetilde{\mathbf{Q}}$ lies the orbit space $\widetilde{\mathbf{C}}$ of the subspace $\mathbf{C}$ of all quadratic systems with a center. Since the singularities with at least one zero eigenvalue, of the quadratic vector fields cannot be centers $([4,43,17])$, we may assume the systems to be of the form (3.S) or $\left(3 . S^{\prime}\right)$ and we only need to consider the group action on such systems $\left(3 . S^{\prime}\right)$. The space $\widetilde{\mathbf{C}}$ is the union of the orbits under the group action of systems of type (III), (II), (I), and (H) of Theorem 3.1.

Since the group of homotheties of the plane acts on the systems $\left(3 . S^{\prime}\right)$, the parameters $a, b, d, A, C$ are homogeneous coordinates of the system $\left(3 . \mathrm{S}^{\prime}\right)$ in the projective space $P_{4}(\mathbb{R})$ where the bifurcation diagrams corresponding to conditions (I), (II), and (III) lie.

The bifurcation diagram corresponding to condition (III). The systems here are of the form $\left(3 . \mathrm{S}^{\prime}\right)$ with $b+d=0$ and they form a hyperplane in $P_{4}(\mathbb{R})$. In [10], Frommer observed that the condition $a+c=0=b+d$ is preserved under a rotation of axes. After performing a rotation of axes of an angle $\theta$, the new coefficient $a^{\prime}$ of $x^{2}$ in the second equation of (3.S) becomes

$$
a^{\prime}=a \cos ^{3} \theta+(3 b+\alpha) \cos ^{2} \theta \sin \theta+(3 c+\beta) \cos \theta \sin ^{2} \theta+d \sin ^{3} \theta .
$$

Thus, if $a \neq 0$, we may always find $\theta$ such that $a^{\prime}=0$. Hence to draw the bifurcation diagram of the orbit space associated with the group action for the case (III) it suffices to consider systems $\left(3 . S^{\prime}\right)$ with $a=0$

$$
\frac{d x}{d t}=-y-b x^{2}-C x y-d y^{2}, \quad \frac{d y}{d t}=x+A x y
$$

with $b+d=0$. These systems form a plane parametrized by $b, C, A$ one of them being nonzero for a nonlinear system. A similarity may be used to render one of the parameters equal to one. We first consider the case $C \neq 0$. These form an affine plane parametrized by $(b / C, A / C)$ or assuming $C=1$, by $(b, A)$. The bifurcation diagram in this case is drawn in the affine plane $(b, A)$. 
A particular integral of a system (3.1) (which could be real or complex), is a curve $F(x, y)=0$ such that for all the points on this curve we have

$$
\frac{\partial F}{\partial x}(x, y) f(x, y)+\frac{\partial F}{\partial y}(x, y) g(x, y)=0 .
$$

For real systems, in the literature sometimes the term invariant curve is used. To be precise we call invariant set of a real system (3.1) a subset $M$ of $\mathbb{R}^{2}$ such that for every initial condition $\left(t_{0}=0 ; x_{0}, y_{0}\right)$, such that $\left(x_{0}, y_{0}\right) \in M$, if $(x(t), y(t)), t \in\left(\alpha_{0}, \beta_{0}\right)$, is the unique maximal solution corresponding to this initial condition, then $(x(t), y(t))$ belongs to $M$ for all $t$ in its maximal interval of definition $\left(\alpha_{0}, \beta_{0}\right)$. Clearly a (nonempty) real curve $F(x, y)=0$ is invariant for (3.1) if and only if it is a particular integral of the system and for real systems, the two terms will be used synonymously.

For $A \neq 0$, the systems (4.1) have the invariant straight line $1+A y=0$. For a system (3.S) the origin is either a center or a focus so an invariant straight line cannot pass through the origin and hence it can be assumed to be of the form

$$
s x+l y+1=0 .
$$

Since this line is invariant, for every point on this line we must have $s P(x, y)+$ $l Q(x, y)=0$ where $P, Q$ denote the polynomials on the right-hand side of (3.S). This is the equation of a conic curve which must have a component in common with (4.2), i.e., we must have an identity

$$
s P(x, y)+1 Q(x, y)=(s x+l y+1)(m x+n y)
$$

for some $m, n$. Straightforward calculations yield:

Proposition 4.1. (i) A system (3.S') has a straight line (4.2) satisfying an identity (4.3) if and only if $b+d=0$ or else $a=0 \neq A$. In the first case the system has at most three such straight lines. If $b+d \neq 0$, the straight line is unique and its equation is $1+A y=0$. (ii) $A$ real quadratic system with a weak focus $F$ and a real invariant straight line has either a center or a weak focus of order one at $F$.

Remark 4.1. Clearly, the presence of two real invariant straight lines of a real system $\left(3 . \mathrm{S}^{\prime}\right)$, forces the origin to be a center.

Corollary 4.1. A real quadratic system (3.S') with a center at the origin and which has an invariant straight line satisfies either the condition (III) or (II).

The basic features of the phase portraits for the case (III) are: the invariant straight lines, the singular points of the systems in the affine plane $(x, y)$ and the singular points at infinity. We also have first integrals for case (III) listed in Table 1.

We give the results of the calculations for these basic features of the phase portraits for case (III). The generic case turns out to be the case when $b A C \Delta(A+b) \neq 0$, where $\Delta=C^{2}+4 b(A+b)$. In this case, for a system (4.1) satisfying (III), we have two possibilities:

(i) If $\Delta<0$ we have only one invariant straight line $L_{A}: 1+A y=0$. (ii) If $\Delta>0$ then we have three straight lines as particular integrals, i.e., the line $L_{A}: 1+A y=0$ and the lines

$$
L_{+}, L_{-}:(C \pm \sqrt{\Delta}) x / 2-b y+1=0 .
$$


These three lines intersect at the singular points.

$$
P_{+}, P_{-}=((C \pm \sqrt{\Delta}) / 2 A b,-1 / A) \quad \text { and } \quad P_{b}=(0,1 / b)
$$

where $P_{+} \in L_{A} \cap L_{-}, P_{-} \in L_{A} \cap L_{+}$, and $P_{b} \in L_{+} \cap L_{-}$. The points at infinity of the invariant lines $L_{A}, L_{+}, L_{-}$are singular points at infinity of the systems: $P_{\infty A}, P_{\infty+}, P_{\infty-}$ where

$$
P_{\infty A}=(z=0, u=0) \text {, and } P_{\infty+}, P_{\infty-}=(z=0, u=(C \pm \sqrt{\Delta}) / 2 b) .
$$

For the study of the singular points at infinity we use the variables $z, u$ with the change $z=1 / x, u=y / x$, and $z, v$ with the change $z=1 / y, v=x / y$ corresponding to the needed two charts. After time rescaling $t=z \tau$, the new equations in $(z, u),(z, v)$ are respectively:

$$
\begin{gathered}
\frac{d z}{d \tau}=z\left(u z+b+C u-b u^{2}\right), \\
\frac{d u}{d \tau}=z\left(1+u^{2}\right)+u\left[(A+b)+C u-b u^{2}\right], \\
\frac{d z}{d \tau}=-z v(z+A), \quad \frac{d v}{d \tau}=-z\left(1+v^{2}\right)-(A+b) v^{2}-C v+b .
\end{gathered}
$$

For the three singular points (4.6) we have real eigenvalues whose nature is given by their products

$$
\begin{gathered}
\left(\lambda_{1} \cdot \lambda_{2}\right)_{\infty A}=b(A+b), \\
\left(\lambda_{1} \cdot \lambda_{2}\right)_{\infty+}=A \sqrt{\Delta}(\sqrt{\Delta}+C) / 2 b, \quad\left(\lambda_{1} \cdot \lambda_{2}\right)_{\infty-}=A \sqrt{\Delta}(\sqrt{\Delta}-C) / 2 b .
\end{gathered}
$$

We sum up the main features of the phase portraits for case (III) in the following two propositions.

Proposition 4.2. Consider system (4.1) with $b+d=0 \neq b A C \Delta(A+b)$. We may suppose $C=1$. We have

If $A b>0$, then $\Delta>0$ and the three singular points $P_{+}, P_{-}, P_{b}$ are all ordinary saddles while the singular points at infinity are all nodes. If $A b<0$ we distinguish two cases: $b(A+b)>0$ and $b(A+b)<0$.

(i) If $b(A+b)>0$ then $\Delta>0, P_{+}$and $P_{-}$are nodes, $P_{b}$ is a saddle while $P_{\infty A}$ is a node and $P_{\infty+}, P_{\infty-}$ are saddles.

(ii) If $b(A+b)<0$ : If $\Delta>0$ then $P_{+}, P_{b}$ are nodes and $P_{-}$is a saddle; $P_{\infty A}, P_{\infty+}$ are saddles and $P_{\infty-}$ is a node. If $\Delta<0$ then we have only one nonzero singular point $P_{b}$ which is a focus and only one point at infinity $P_{\infty A}$ which is a saddle.

For the nongeneric case (III) we have

Proposition 4.3. Consider a system (4.1) with $b+d=0=b A \Delta(A+b), C \neq 0$ (we may assume $C=1$ ). We have:

If $\Delta=0$ then $b(A+b)<0, A b<0, P_{+}=P_{-}$is a saddle-node and $P_{b}$ is a node. $P_{\infty A}$ is a saddle, $P_{\infty+}=P_{\infty-}$ is a saddle-node.

If $A+b=0 \neq b$ then $A b<0, P_{-}=P_{b}$ is a saddle-node, $P_{+}$is a node, $P_{\infty A}=P_{\infty-}$ is a saddle-node, and $P_{\infty+}$ is a saddle.

If $b=0 \neq A, L_{+}$is $C x+1=0, L_{A} \cap L_{+}=\left\{P_{A+}\right\}$ where $P_{A+}=$ $(-1 / C,-1 / A)$ is a saddle, and $P_{\infty L+}=(z=0, v=0)$ and $P_{\infty A}=(z=$ $0, u=0)$ are saddle-nodes. 
If $A=0 \neq b$ we have $\Delta>0, P_{b}$ is a saddle, $P_{\infty_{+}}, P_{\infty_{-}}$are saddle-nodes and $z=0=u$ is a node.

If $A=0=b$, the system has only one singular point $(0,0)$, one invariant line $L_{+}$and two points at infinity (as well as their opposites): $z=0=v$ which is a degenerated singular point of trace $-C$ and it is a saddle and $z=0=u$ which is a degenerated singularity of trace 0 .

Using all this information, we draw the bifurcation diagram for case (III), with $C \neq 0$. We may assume $C=1$. This bifurcation diagram is drawn in the plane $(b, A)$ and it appears in Figure 1. The bifurcation lines are: the hyperbola $\Delta=0$, the lines $b=0, A=0$, and $A+b=0$. The line at infinity of this affine plane, when completed, is $C=0=a=b+d$ and it also belongs to the bifurcation diagram corresponding to condition (II). We postpone the drawing of the full bifurcation diagram for (III) on a disk representing $P_{2}(\mathbb{R})$ when opposite points of the circumference are identified, until we complete the bifurcation diagram for case (II).

The bifurcation diagram corresponding to case (II), i.e., $a=0=C$. Case (II) is the symmetric case with respect to the $y$-axis:

Proposition 4.4. $A$ vector field $\left(3 . S^{\prime}\right)$ is symmetric with respect to the $y$-axis if and only if it satisfies $a=0=C$.

The systems satisfying condition (II) are of the form

$$
\frac{d x}{d t}=-y-b x^{2}-d y^{2}, \quad \frac{d y}{d t}=x+A x y .
$$

We calculate the basic features of the phase portraits in this case. By Proposition 4.1 if $A(b+d) \neq 0$, such a system has a unique invariant straight line $L_{A}: 1+A y=0$. Assuming $A b d(A-d) \neq 0$ and $b(A-d)>0$ then the system has three nonzero singular points:

$$
P_{d}=(0,-1 / d), \quad P_{+}, P_{-}=\left( \pm \sqrt{A-d} / A^{2} b,-1 / A\right) .
$$

We note that $P_{+}, P_{-}$are located on $L_{A}$. The eigenvalues of $P_{d}$ are

$$
\lambda_{1 d}, \lambda_{2 d}= \pm \sqrt{d-A} / d
$$

and those of $P_{+}, P_{-}$are

$$
\lambda_{1 \pm}=\mp 2 b \sqrt{A-d} / A^{2} b, \quad \lambda_{2 \pm}= \pm A \sqrt{A-d} / A^{2} b .
$$

As before, to determine the behaviour at infinity we use the variables $z, u$ and $z, v$ and after time rescaling $t=z \tau$ we obtain the equations:

$$
\begin{aligned}
& \frac{d z}{d \tau}=z\left(u z+b+d u^{2}\right), \quad \frac{d u}{d \tau}=z\left(1+u^{2}\right)+u\left(A+b+d u^{2}\right) \\
& \frac{d z}{d \tau}=-z u(z+A), \quad \frac{d v}{d \tau}=-z\left(1+v^{2}\right)-\left((A+b) v^{2}+d\right)
\end{aligned}
$$

The singular points come in opposite pairs and in the variables $z, u$ they are

$$
P_{\infty A}: \quad z=0=u, \quad P_{\infty \pm}: \quad z=0, u= \pm \sqrt{-(A+b)} / d .
$$

The eigenvalues of these points are respectively:

$$
\lambda_{1 A}=b, \quad \lambda_{2 A}=A+b ; \quad \lambda_{1 \pm}=-A, \quad \lambda_{2 \pm}=-2(A+b) .
$$




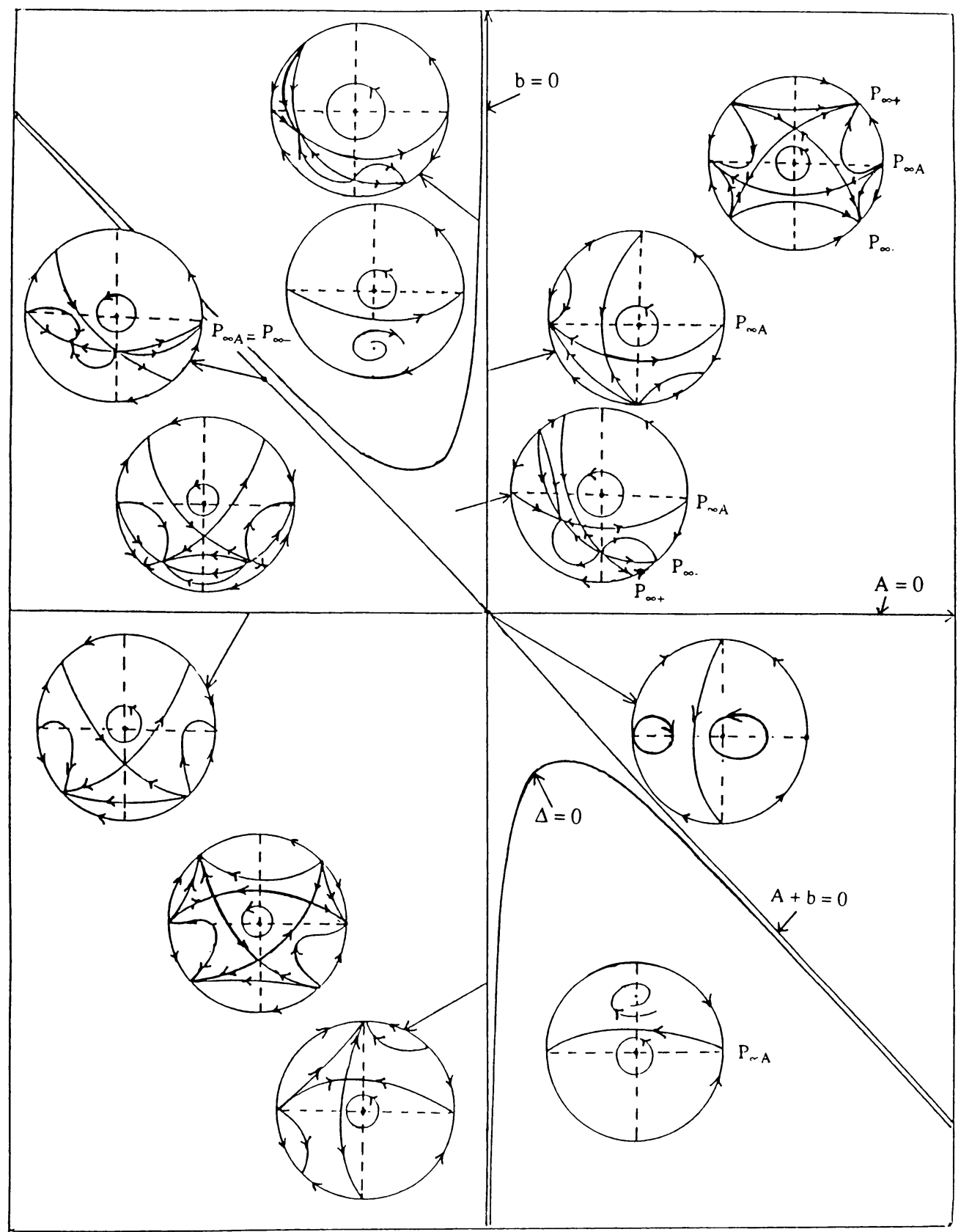

FIgURE 1

The constant of motion in case (II) in the generic case $A b d(A+b)(A+2 b) \neq 0$ is:

(4.18)

$\left\{b(A+b)(A+2 b) x^{2}-(A+b-d)+2 b(A+b-d) y+b d(A+2 b) y^{2}\right\}|1+A y|^{2 b / A}$

We observe that this constant of motion is analytic for $1+A y \neq 0$. From (4.18) we see that the system has a conic particular integral obtained by annihilating the first factor in (4.18). 
We give below the essential features of the phase portraits for this generic case (II).

Proposition 4.5. Consider a system (4.10) in the generic case

$$
\operatorname{Abd}(A+b)(b+d)(A-d)(A+b-d) \neq 0 .
$$

We may assume for simplicity that $d>0$. Then we have:

(i) The system has four singular points iff $b(A-d)>0$. If $b>0$ and $A-d>0$, then we have two saddles: $P_{+}, P_{-}$and two centers. If $b<0$ and $A-d<0, P_{d}$ is a saddle and $P_{+}, P_{-}$are nodes when $A>0$ while $P_{+}, P_{-}$ are saddles if $A<0$. If $b(A-d)<0$, we have only one nonzero singular point: $P_{d}$ which is a center if $A-d>0, b<0$ and it is saddle if $A-d<0, b>0$.

(ii) If $A+b<0$ we have three pairs of (opposite) singular points at infinity. $P_{\infty A}$ is a node for $b(A+b)>0$ and a saddle for $b(A+b)<0, P_{\infty+}, P_{\infty-}$ are nodes if $A(A+b)>0$ and saddles if $A(A+b)<0$. If $A+b>0$ we have only one pair of (opposite) singular points at infinity. If $b>0, P_{\infty A}$ is a node and it is a saddle if $b<0$.

(iii) If $A+b<0$, the conic integral of the system is a hyperbola. If $A+b>0$, the conic integral is an invariant ellipse if $(A+b-d)(b+d) b>0$ and it is $a$ conic which has only complex points, if $(A+b-d)(b+d) b<0$.

The nongeneric cases satisfying (II), i.e., those with

$$
\operatorname{Abd}(A-d)(A+b)(A+2 b)=0=a=C
$$

are treated (analogously with our discussion for condition (III)), using the information contained in equations (4.11)-(4.18) and in Table 2.

The bifurcation diagram for the case $d \neq 0$ (we may assume $d=1$ ) appears in Figure 2, except for the orientation of some integral curves, omitted not to overcrowd the drawing. To follow the change in the systems as parameters vary, we drew all the phase portraits on this bifurcation diagram and to make this possible, we varied the scale when drawing our disks. Condition (II) with $d \neq 0$ defines an affine plane which is parametrized by $(b / d, A / d)$ or if we assume $d=1$, by $(b, A)$. The bifurcation points are located on the lines $b=0$, $A-d=0, A+b=0, A=0$ and on the line $b+d=0$. We complete the bifurcation diagram for case (II) by considering what happens in the case $d=0$. The bifurcation diagram for the line $a=0=c=C=d, A=1$, line parametrized by $b$, is drawn in Figure 3 (page 816); the bifurcation points are at $b=-A$ and at $b=0$. The point at infinity of this line is the point $C=a=A=d=0 \neq b$. The system corresponding to this point has only one singular point, the origin and the phase portrait is given in Figure 4 . The full bifurcation diagram for case (II) is drawn on a disk. Inside the disk we place the bifurcation diagram shown in Figure 2 and on the circle the bifurcation diagram shown in Figure 3 and completed with its point at infinity for which we have the phase portrait given in Figure 4 (page 816 ). The complete diagram for condition (II) appears on the disk shown in Figure 5 (page 817) where almost all the phase portraits are drawn; the missing ones in the central quadrangle and the orientation of the some of the integral curves could be found in Figure 2.

Finally, we are now in a position to complete on the disk the bifurcation diagram for case (III). We place inside the disk the bifurcation diagram for the plane $b+d=0=a, C \neq 0$, drawn in Figure 1 and on the circle the bifurcation 


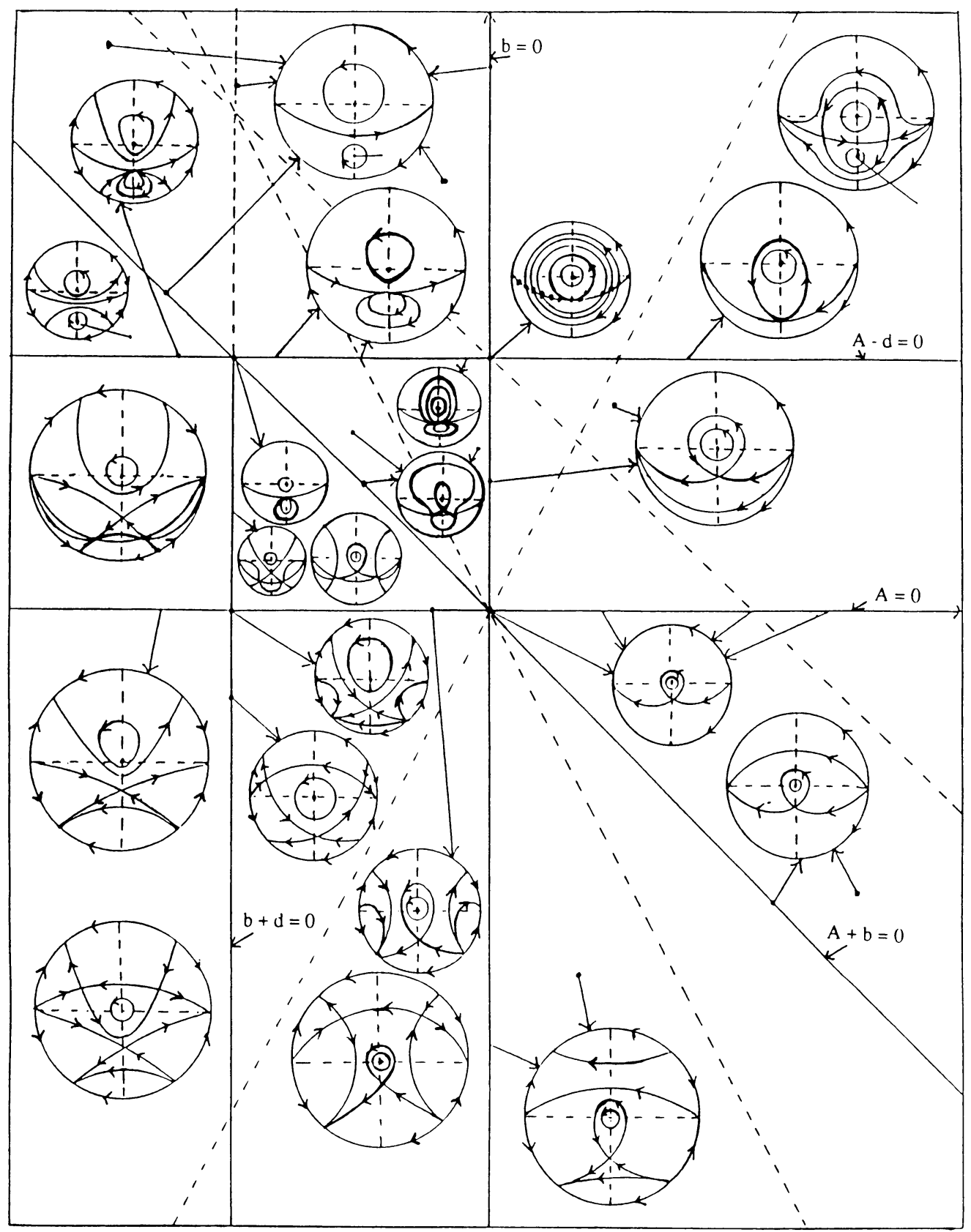

Figure 2

diagram for the line $a=0=C=b+d$, which appears in Figure 5. The full diagram for case (III) appears in Figure 6 (see page 818).

Remark 4.2. We remark that the system $d x / d t=-y, d y / d t=x+A x y$ which satisfies conditions (III) and (II) can be brought to the form $d X / d t=$ $-Y-A X Y, d Y / d t=X$ by using the transformation $X=y, Y=-x$. The portrait of the first system is situated on the circumference of the disk 


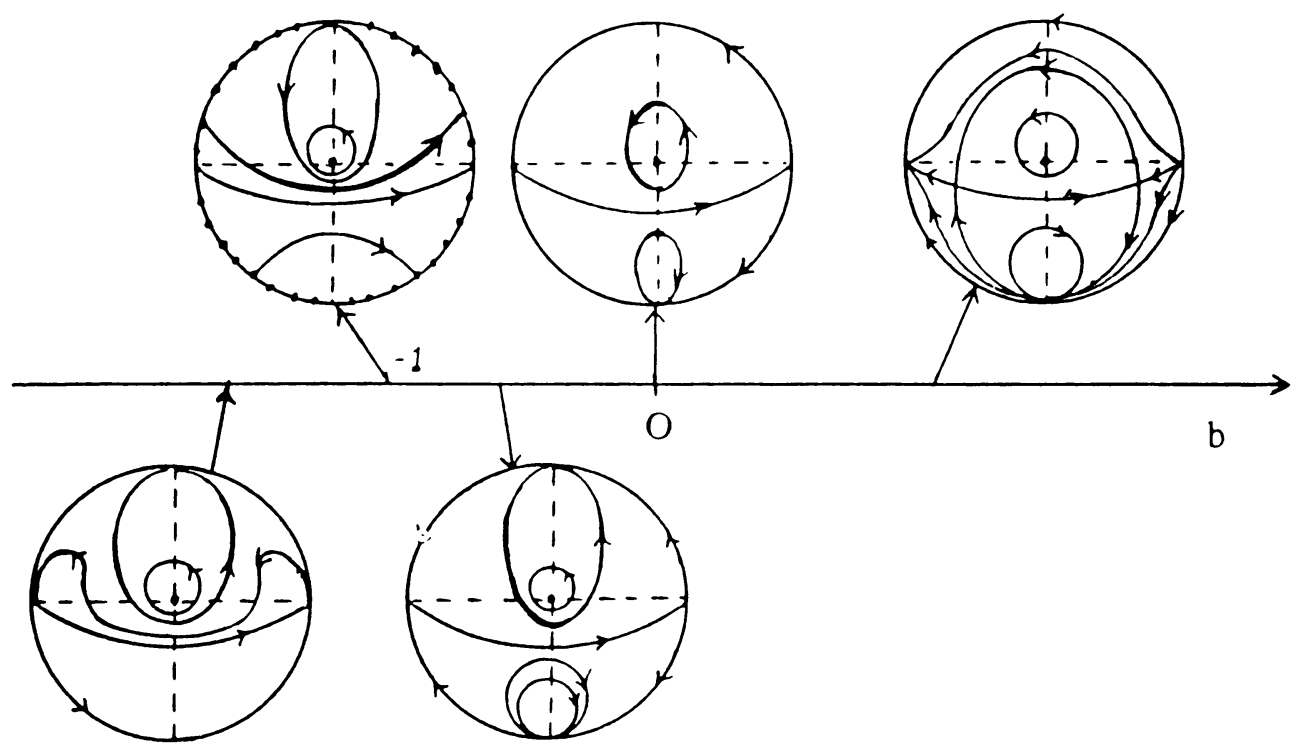

FIGURE 3

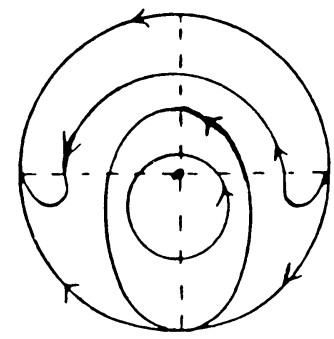

Figure 4

in Figure 6 and corresponds to the point $a=C=b=d=0 \neq A$. The phase portrait of the second system is situated at the center of the circle (i.e., $b=0=d=a=A \neq C=1)$. Thus additional identifications are needed in order to obtain the orbit space of the systems corresponding to condition (III) under the group action.

The bifurcation diagram for case (I): $C+2 a=0=A+3 b+5 d=a^{2}+2 d^{2}+b d$. The algebraic variety determined by these equations is the conic $a^{2}+2 d^{2}+b d=$ 0 , situated on the projective plane determined by the equations $C+2 a=0$ and $A+3 b+5 d=0$. We may parametrize this plane by the homogeneous coordinates $a, b, d$. We can draw the bifurcation diagram of the systems (I) on the curve $a^{2}+2 d^{2}+b d=0$ in this projective plane which we can represent on the disk, with $a=0$ corresponding to the circumference. In the affine plane associated with the chart $U_{a}(a \neq 0)$, this conic is a hyperbola. If $a \neq 0$, since $a^{2}+2 d^{2}+b d=0$, we must have $d \neq 0$. Hence $b=-\left(2 d^{2}+a^{2}\right) / d$ and the system is:

$\frac{d x}{d t}=-y+\frac{\left(2 d^{2}+a^{2}\right) x^{2}}{d}+2 a x y-d y^{2}, \quad \frac{d y}{d t}=x+a x^{2}-\frac{\left(d^{2}+3 a^{2}\right) x y}{d}-a y^{2}$. 


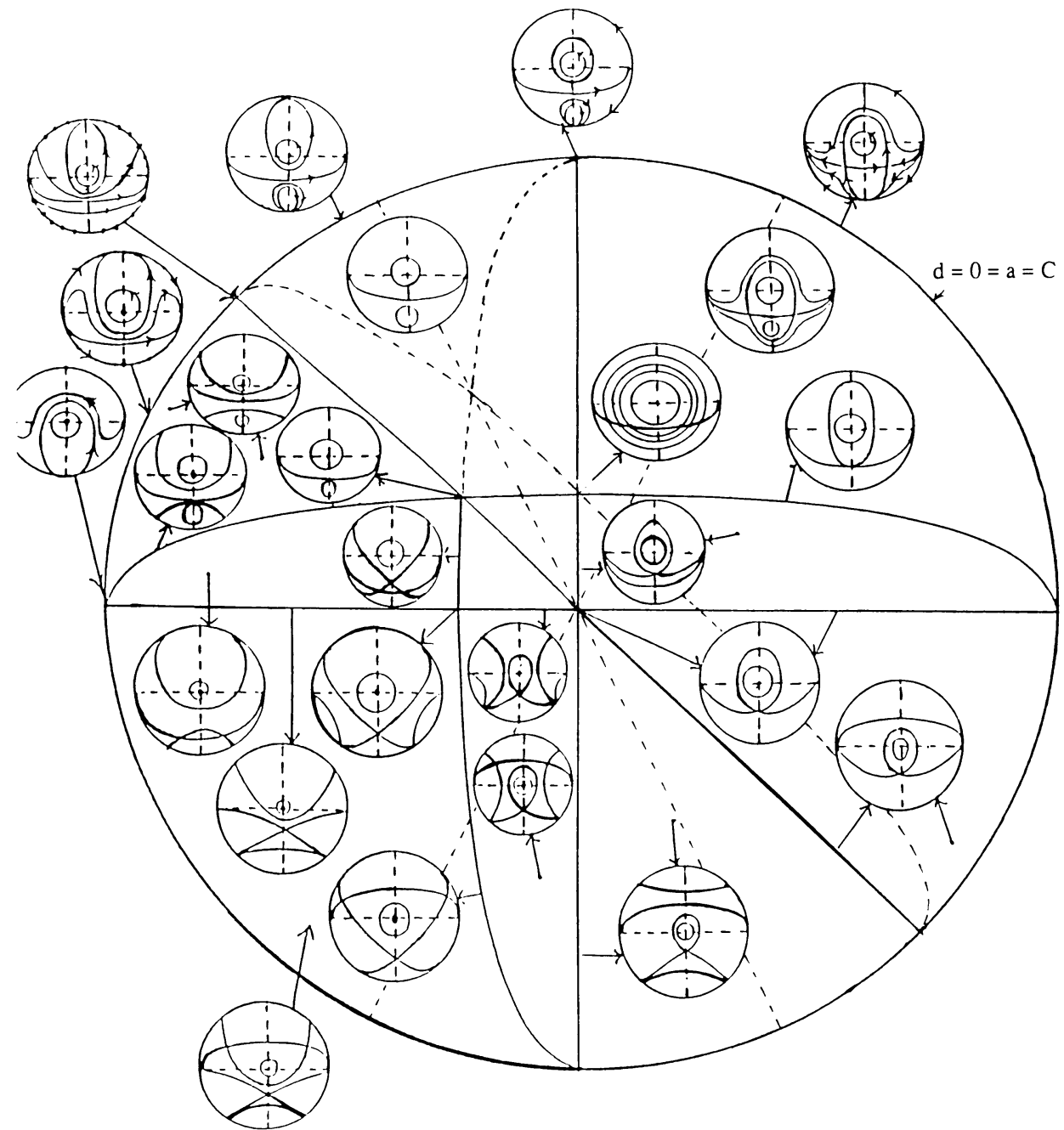

FIGURE 5

We may assume $a=1$. To study the singular points and the behaviour at infinity, we change coordinates as in [8]: $X=-a x+d y, Y=d y$. The equations in $X, Y$ yield:

$$
\frac{d Y}{d X}=\frac{\lambda X-\lambda Y-\lambda X^{2}+3 X Y-2 Y^{2}}{\lambda X-Y+X^{2}-X Y}
$$

where $\lambda=d^{2} /\left(a^{2}+d^{2}\right)$. We first observe that on $X+1=0$, there is no singular point of the equation. The equation $-X Y+X^{2}-Y+\lambda X=0$ gives $Y=X(X+\lambda) /(X+1)$. Substituting this into $\lambda X-\lambda Y-\lambda X^{2}+3 X Y-2 Y^{2}=0$ for $X \neq 0$, we obtain $X^{3}+3 X^{2}+3 \lambda X+\lambda=0$. This cubic equation has the discriminant $-108 \lambda\left(\lambda^{2}-\lambda+1\right)$, which is negative. Hence we have only one nonzero singular point. This point turns out to be a node for all $d, d \neq 0$ (cf. [37]). To study the points at infinity we use the change $z=1 / x, u=y / x$ and $z=1 / y, v=x / y$ and after time rescaling $t=\tau z$, we obtain the equations:

$$
\frac{d z}{d \tau}=z(-\lambda z-1+u+u z), \quad \frac{d u}{d \tau}=-\lambda+2 u-u^{2}+z\left(\lambda-2 \lambda u+u^{2}\right)
$$




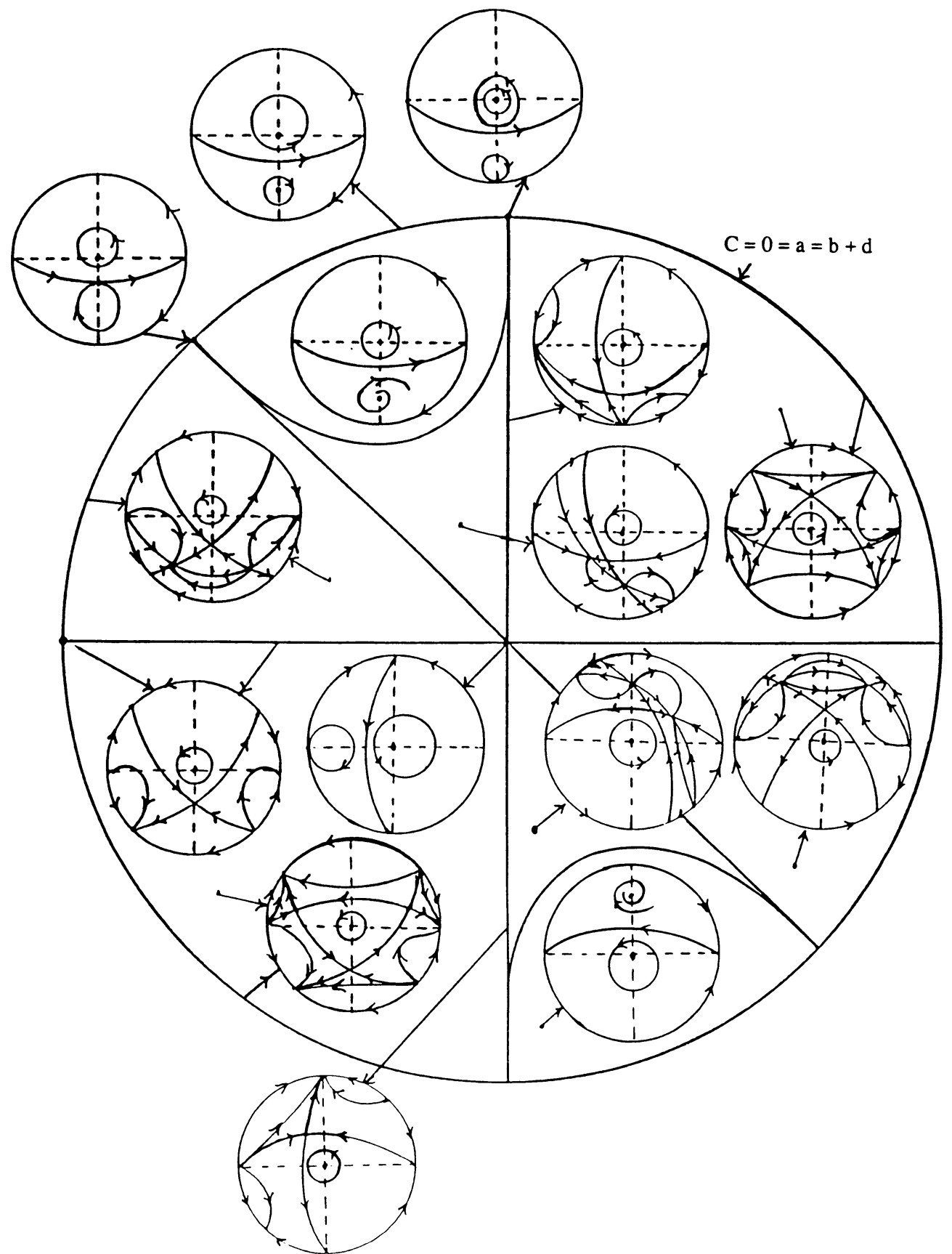

Figure 6

$$
\begin{aligned}
& \frac{d z}{d \tau}=z\left(-\lambda z v+\lambda z+\lambda v^{2}-3 v+2\right), \\
& \frac{d v}{d \tau}=z\left(2 \lambda v-1-\lambda v^{2}\right)+v\left(-2 v+\lambda v^{2}+1\right) .
\end{aligned}
$$

Hence for all $d \neq 0$, the systems have three pairs of opposite singular points at infinity. On the two charts the points at infinity are: $z=0=v$ which is a 


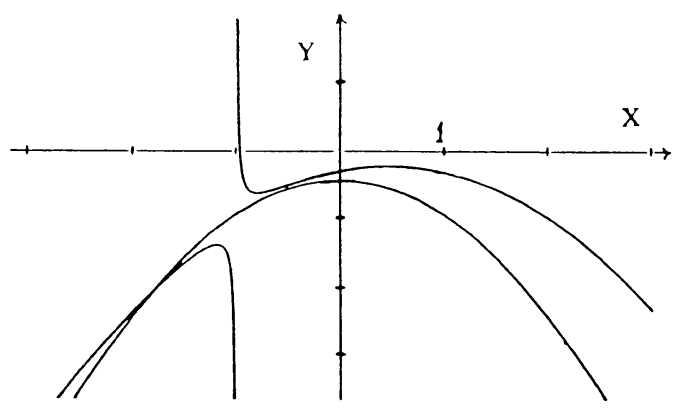

FIGURE 7

node and $z=0, u=1 \pm \sqrt{1-\lambda}$ which are saddles.

The general integral for systems $\left(3 . \mathrm{S}^{\prime}\right)$ satisfying (I), with $a \neq 0$, was calculated by Ye Yan-Qian (cf. [45] or [37]) following Dulac [9] and it is

$$
\begin{gathered}
\left\{d^{2}+3 d\left(d^{2}+a^{2}\right) y+3 d^{2}\left(d^{2}+a^{2}\right) y^{2}\right. \\
\left.-3 a d\left(d^{2}+a^{2}\right) x y+\left(d^{2}+a^{2}\right)(d y-a x)^{3}\right\}^{2} \\
=D\left[d^{2}+2 d\left(d^{2}+a^{2}\right) y+\left(d^{2}+a^{2}\right)(a x-d y)^{2}\right]^{3} .
\end{gathered}
$$

From this general integral, we see that we have two invariant algebraic curves, a conic which is a parabola and a cubic curve. The two curves interest at a point in the $(X, Y)$ plane and they are drawn (for $\lambda=8 / 9)$ in Figure 7. They also intersect at infinity where the cubic has a single point coinciding with the point at infinity of the parabola. The picture of the curves, including the points at infinity, appears on the disk drawn in Figure 8 (next page). For case (I) formula (4.23) yields a rational function as a constant of motion:

$$
\frac{\left\{d^{2}+3 d\left(d^{2}+a^{2}\right) y+3 d^{2}\left(d^{2}+a^{2}\right) y^{2}-3 a d\left(d^{2}+a^{2}\right) x y+\left(d^{2}+a^{2}\right)(d y-a x)^{3}\right\}^{2}}{\left[d^{2}+2 d\left(d^{2}+a^{2}\right) y+\left(d^{2}+a^{2}\right)(a x-d y)^{2}\right]^{3}}
$$

which is undefined on the conic curve obtained by annihilating the denominator. Using this information for the systems $\left(3 . S^{\prime}\right)$ satisfying (I) with $a \neq 0$, we obtain the phase portrait for this case, which is indicated in Figure 9 (next page).

The system (3.S') satisfying condition (I) with $a=0$ also satisfy condition (II), already discussed and in this case either $d=0$ or $b+2 d=0$. The full bifurcation diagram for condition (I) is drawn in Figure 10 (page 821) where on the hyperbola inside the circe we have the phase portrait in Figure 9.

The bifurcation diagram corresponding to the disjunction of conditions (I), (II), (III), and (H) lies in $P_{4}(\mathbb{R})$. The full picture lies in the union in $P_{4}(\mathbb{R})$ of the two hyperplanes $C+2 a=0$ and $a=0$. We represent a hyperplane in $P_{4}(\mathbb{R})$ as a three-dimensional ball with opposite points on the sphere identified. In Figure 11 (page 821) we have the hyperplane $C+2 a=0$ where on the sphere we placed the plane $\mathbf{C}_{\mathrm{II}}$ and where inside the sphere we drew $\mathbf{C}_{\mathrm{I}}, \mathbf{C}_{\mathrm{H}}$, as well as the plane cut by $\mathbf{C}_{\mathrm{III}}$ on the hyperplane $C+2 a=0$. A similar ball represents the hyperplane $a=0$ where we place on the sphere the plane $C=0$ which is $\mathbf{C}_{\mathrm{II}}$ and where only two of the points of $\mathbf{C}_{\mathrm{I}}$ appear. 


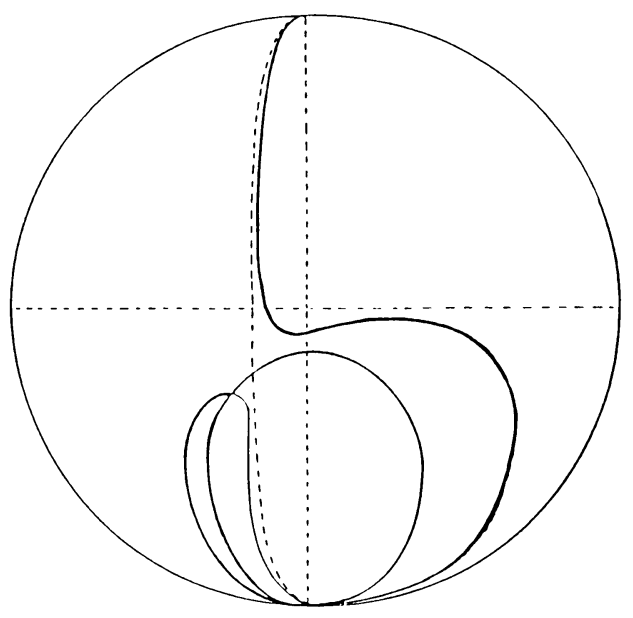

FIGURE 8

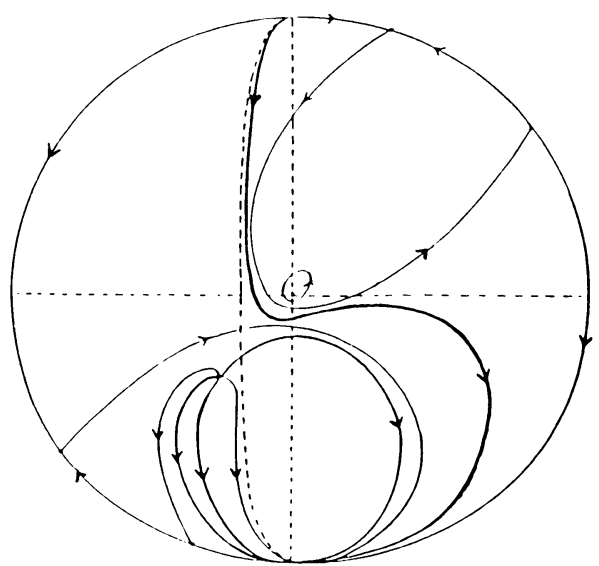

Figure 9

5. THE KEY FEATURES OF THE QUADRATIC SYSTEMS WITH A CENTER: THE LOW ORDER ALGEBRAIC CURVES, PARTICULAR INTEGRALS OF THE SYSTEMS

For quadratic systems which are Hamiltonian, in particular for systems in $\mathbf{C}_{\mathrm{H}}$, the integral curves lie on cubic curves, level curves of the cubic Hamiltonian. For quadratic systems $\left(3 . S^{\prime}\right)$ with a center satisfying one of the conditions (III), (II), (I) we also have algebraic curves which are key features of the phase portraits of the systems.

In the generic cases (III) and (II) we have a straight invariant line. Furthermore, in case (III) we have two other straight lines $L_{+}, L_{-}$(real or complex conjugate) of the form (4.2) and satisfying equation (4.3). We may also form the real conic curve having $L_{+}, L_{-}$as components. For the generic case (II), the zero level curve of the first integral is a conic curve depending on the parameters $b, d$, and $A$. Thus we construct the following algebraic curves which are particular integrals (for a formal definition, see $\S 6$ ) of the systems corresponding 


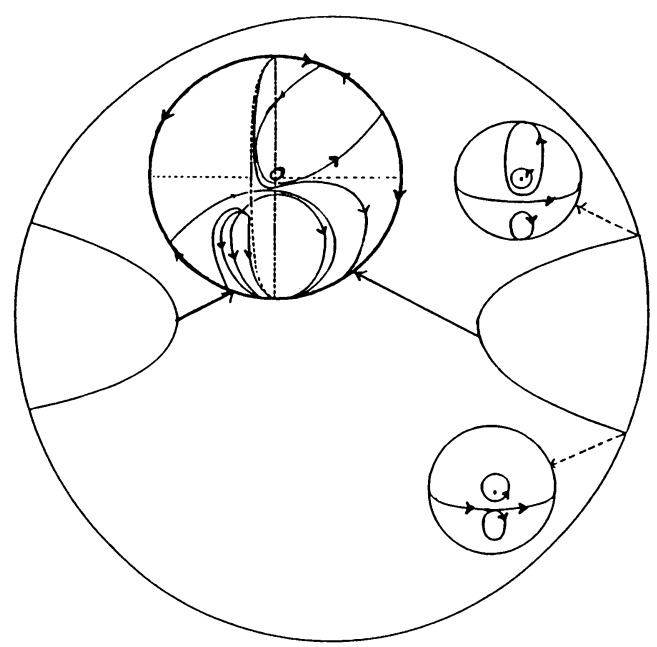

FIGURE 10

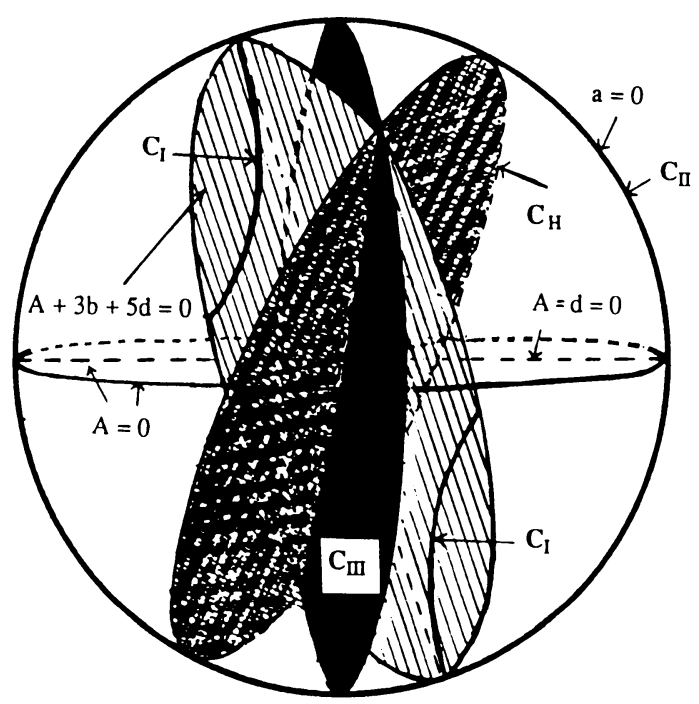

FIGURE 11

to the cases (III) and (II) respectively:

$$
\begin{aligned}
(1+A y)\left\{(C x / 2-b y+1)^{2}\right. & \left.-\left(C^{2}+4 b(A+b)\right) x^{2} / 4\right\}=0, \\
(1+A y)\left\{b(A+b)(A+2 b) x^{2}\right. & +b d(A+2 b) y^{2} \\
& +2 b(A+b-d) y-(A+b-d)\}=0,
\end{aligned}
$$

We note that in the generic case, the conic component of (5.2) is an irreducible curve.

In case (I), if $a \neq 0$, we must have $d \neq 0$ and using the equations for (I), we can eliminate the parameters $A$ and $C$ and obtain the general integral (4.23) which yields two invariant algebraic curves, a parabola and a cubic curve.

We discuss the role played by these algebraic curves in the change of the phase portraits as parameters vary. For a family of conic curves (respectively 
cubic curves) depending on parameters, a point in the parameter space will be called a bifurcation point if any neighborhood of this point contains points with conics (respectively cubic curves) of different affine types. We use the term "bifurcation" in the sense employed by Arnold (cf. [2, p. 210]) to indicate any qualitative, topological modification obtained for the objects we consider (which may be algebraic curves or surfaces, plane vector fields, etc.) as a consequence of the variation of the parameters. The objects in our case are affine algebraic curves which will be considered together with their projective completion.

We have:

Theorem 5.1. Consider the bifurcation diagram of the real system $\left(3 . \mathrm{S}^{\prime}\right)$ satisfying one of the conditions (III), (II), (I). We have:

(i) For case (III) it suffices to consider $a=0$. The bifurcation diagram of the systems $\left(3 . \mathrm{S}^{\prime}\right)$ satisfying the condition $b+d=0=a, C \neq 0$, coincides with the bifurcation diagram of the associated family of affine cubic curves (5.1).

(ii) Each bifurcation point of the systems $\left(3 . \mathrm{S}^{\prime}\right)$ verifying condition (II) is also a bifurcation point for the family of curves (5.2). (iii) The bifurcation points of the systems $\left(3 . \mathrm{S}^{\prime}\right)$ satisfying condition (I) are bifurcation points for the family of invariant cubic curves of the systems satisfying this condition.

Proof. (i) For condition (III) we consider the algebraic variety defined by the equations $a=0=b+d$. This is a projective plane with homogeneous coordinates $(b, A, C)$. For $C \neq 0$, the bifurcation lines are: the conic $\Delta=0$ (which is a hyperbola in the affine part corresponding to $C \neq 0$ ) and the lines $b=0, A=0, A+b=0$. These are exactly the bifurcation lines of the family of the affine curves $(5.1)$ in this case. Indeed, on $\Delta=0$, the conic component of this cubic is the double line $(C x / 2-b y+1)^{2}=0$. On $b=0$ the conic is reducible over the reals, formed by the straight line $C x+1=0$ and the line at infinity. On $A=0$ the straight line component becomes the line at infinity and on $A+b=0$, the conic component of (5.1) is reducible and formed by the line $C x-b y+1=0$ and by the component $1+A y=0$ of (5.1). The cubic curve (5.1) can be viewed as three straight lines. On the bifurcation lines, two of these straight lines coincide or one of them becomes the line at infinity. We see that we have the same bifurcation lines for both the phase portraits and the family of cubics (5.1).

(ii) Condition (II): $a=0=C$ defines a projective plane with homogeneous coordinates $(b, A, d)$. All bifurcation points for the systems $\left(3 . \mathrm{S}^{\prime}\right)$ satisfying condition (II) are also bifurcation points for the family of affine cubic curves (5.2). Indeed, the bifurcation points for condition (II) are located on the lines $A=0, b=0, A-d=0, A+b=0$, and $b+d=0$ and on $d=0$, drawn in Figure 5, the last one being the circumference. The bifurcation diagram of the family of curves (5.2) with $d=$ constant, is formed by the lines: $A=0$, where the straight line component becomes the line at infinity; $b=0$ where the conic component of (5.2) becomes the double line at infinity; $A-d=0$ where the cubic (5.2) is singular and the straight line component of this cubic is tangent to the conic component; $A+b=0$ where the cubic (5.2) becomes the triple line $(1+A y)^{3}=0 ; A+b-d=0$ where for $B \neq 0$, the conic component becomes the reducible conic $x^{2}+y^{2}=0 ; A+2 b=0$ where the conic component of (5.2) becomes reducible, formed by the line at infinity and the line $A y+1=0$; the line $d=0=a=C$ is also a bifurcation line for 


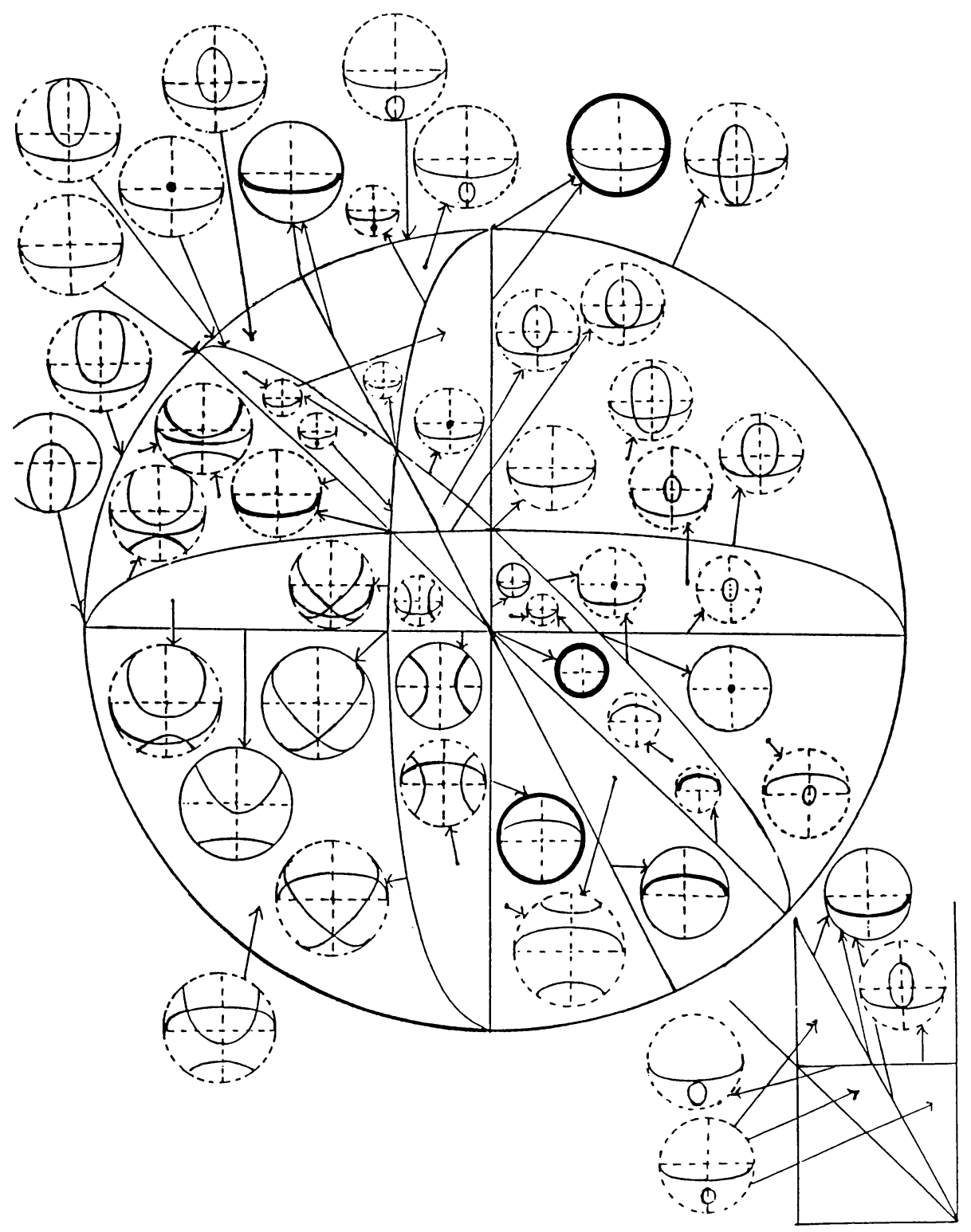

FIGURE 12

(5.2) since on this line the conic component changes its affine type in the affine plane $(x, y)$ and it becomes a parabola. We draw in Figure 12 the bifurcation diagram for the family of curves (5.2), the part corresponding to the central polygon in the disk, appears in the enlargement in the lower right side of Figure 12. The more pronounced lines in Figure 12 represent double or triple lines. Not all the changes in the curves generate a change in the phase portraits but all the changes in the phase portraits occur when we also have a change in the type of the curves.

(iii) The first two conditions, i.e., $A+3 b+5 d=0=C+2 a$ of (I) determine a projective plane with homogeneous coordinates $(b, d, a)$. The affine part, 
for $a \neq 0$, of the curve $a^{2}+2 d^{2}+b d=0$, determined by the third condition in (I) is a hyperbola. This conic cuts the line $a=0$ of this plane at the points $(b, 0,0)$ and $(-2 d, d, 0)$, both of them situated on the bifurcation diagram for case (II). If $a \neq 0$, then all the phase portraits are of one type, namely the one indicated in Figure 9. So the bifurcation diagram for condition (I) are exactly the two points mentioned above and these are also points of bifurcation for the invariant cubic curves in this case. Indeed, for $a \neq 0$ the invariant cubics are irreducible, while for $a=0$ they are reducible curves.

Remark 5.1. The restriction $C \neq 0$ imposed in point (i) of the preceding theorem is necessary there because in (i) we consider only case (III). The points of the line $C=0=b+d=a$, located on the circumference of the disk in Figure 6 , are all bifurcation points for the systems $\left(3 . S^{\prime}\right)$ with a center at the origin but only a segment of this line is made of bifurcation points for systems of type (III) while the remaining segment of this line is made of bifurcation points for systems of type (II), segment which also appears in Figure 2. All points of this line are also bifurcation points for the family of cubic particular integrals of the systems $\left(3 . \mathrm{S}^{\prime}\right)$ with a center at the origin.

Remark 5.2. We point out a striking particular instance of change in phase portraits produced by a bifurcation of invariant algebraic curves: Consider the bifurcation line $b+d=0$ in Figure 2, in the part $A<0$. On both the left and right side of this line and on the line, the phase portraits have exactly three singular points in the plane $(x, y)$ which are all saddles and three pairs of opposite singular points at infinity, all nodes. The phase portraits on the left of the bifurcation line have a singular cycle with two singular points, on the bifurcation line they have a singular cycle with three singular points and on the right a singular cycle with just one singular point, i.e., a homoclinic loop. This complete difference in phase portraits is forced by the bifurcation of the invariant hyperbola on both sides of the bifurcation line $b+d=0$, which becomes a pair of intersecting straight lines on $b+d=0$.

\section{Algebraic PARTICULAR INTEgRALS OF POLYNOMIAL VECTOR FIELDS}

The importance of the algebraic particular solutions of polynomial differential equations was first observed by Darboux in [7].

In this paragraph we consider algebraic particular integrals of general polynomial vector fields, but to be specific we illustrate our discussion by obtaining some results for the quadratic systems with a weak focus.

By a particular integral of a real system

$$
\frac{d x}{d t}=P(x, y), \quad \frac{d y}{d t}=Q(x, y)
$$

it is usually meant a (nonempty) real curve $F(x, y)=0$ such that for all the points on this curve we have

$$
\frac{\partial F}{\partial x}(x, y) P(x, y)+\frac{\partial F}{\partial y}(x, y) Q(x, y)=0 .
$$

If the curve is algebraic then $F$ is of the form

$$
F(x, y)=F_{n}(x, y)+F_{n-1}(x, y)+\cdots+F_{1}(x, y)+F_{0}(x, y)
$$


where $F_{i}(x, y)$ is a homogeneous polynomial with real coefficients of degree $i$.

If for some $K(x, y) \in \mathbb{R}[x, y]$ we have

$$
\frac{\partial F}{\partial x} P+\frac{\partial F}{\partial y} Q=F(x, y) K(x, y) ;
$$

then clearly $F(x, y)=0$ is a particular integral of $(6.1)$.

If there exists a curve $F(x, y)=0$ satisfying (6.CT) for some real polynomial $K$, then (6.CT) is a computational tool for the determination of the coefficients of $F$; we shall use it to calculate the conics and cubics, particular integrals of $\left(3 . \mathrm{S}^{\prime}\right)$. Let us denote by $P_{i}, Q_{i}$ the homogeneous polynomials of degree $i$ in $P$, respectively $Q$ of (6.1). To be specific we consider systems (3.S') where we have $P=P_{1}+P_{2}, Q=Q_{1}+Q_{2}$ and the identity (6.CT) is of the following form:

$$
\frac{\partial F}{\partial x} P+\frac{\partial F}{\partial y} Q=F(x, y)(u x+v y+w) .
$$

Our analysis of the quadratic systems with a center gives us:

Proposition 6.1. Assume that a system (3.S') has a center at the origin. Then, generically, this system has either a straight line and a conic curve (not passing through the origin) satisfying an identity $\left(6 . \mathrm{CT}_{2}\right)$ or it has no invariant straight line but it has a conic and a cubic curve, both irreducible over $\mathbb{C}$ as particular integrals or it is a Hamiltonian system. For each one of these particular integrals $F(x, y)=0$ an identity $\left(6 . \mathrm{CT}_{2}\right)$ holds.

Remark 6.1. In the case of a real algebraic curve $F(x, y)=0$, irreducible over $\mathbb{C}$, which has an infinity of points in $\mathbb{R}^{2}$ and which is a particular integral of system (6.1), (6.CT) holds since the curve must have a component in common with $F^{\prime}(x, y)=0$, where $F^{\prime}=(\partial F / \partial x) P+(\partial F / \partial y) Q$. So, for algebraic curves which are irreducible and which have an infinity of points in $\mathbb{R}^{2}$, (6.CT) is equivalent to the real curve being a particular integral for the real system (6.1).

Even if we are primarily interested in real systems, the polynomial identity (6.CT) is useful beyond the calculation of the irreducible real algebraic curves which have an infinite number of points in $\mathbb{R}^{2}$ and which are particular integrals of the systems. Indeed, for example the conic component of (5.2) has no real point, for the parameter values such that $0<A+b<d, A-d>0$, and $b+d<0$. Nevertheless, as seen in $\S 5$, this conic component has its role. So in the final analysis, real curves with only complex points, as well as those with a finite number of real points, need to be considered. What matters is that these curves satisfy an identity (6.CT). So, from now on, we shall use the term "algebraic particular integral", according to the following definition:

Definition 6.1. By an algebraic particular integral of a polynomial system (6.1), real or complex, we shall mean an algebraic curve $F(x, y)=0$, real or complex, such that an identity (6.CT) holds for some polynomial $K(x, y)$ with real or complex coefficients.

For an algebraic curve $F(x, y)=0$ with $F$ of degree $n,\left(6 . \mathrm{CT}_{2}\right)$ yields $n+2$ equations, one for each coefficient of a degree $i$ term, with $0 \leq i \leq$ $n+1$. Identifying the corresponding coefficients of the zero degree terms, we 
get $F_{0} w=0$ and assuming $F_{0} \neq 0$, we have $w=0$. Identifying the coefficients of the degree 1 terms in $\left(6 . \mathrm{CT}_{2}\right)$ we have $u=a_{01}, v=-a_{10}$. Identifying the coefficients of the degree $i$ terms in $\left(6 . \mathrm{CT}_{2}\right)$, with $2 \leq i \leq n$, we have

$$
\left(6 . \mathrm{CT}_{2 ; i, F}\right) \quad \frac{\partial F_{i}}{\partial x} P_{1}+\frac{\partial F_{i}}{\partial y} Q_{1}+\frac{\partial F_{i-1}}{\partial x} P_{2}+\frac{\partial F_{i-1}}{\partial y} Q_{2}=F_{i-1}\left(a_{01} x-a_{10} y\right)
$$

while identifying the coefficients of degree $n+1$ in $\left(6 . \mathrm{CT}_{2}\right)$ we have

$$
\text { (6.CT } 2 ; n+1, F) \quad \frac{\partial F_{n}}{\partial x} P_{2}+\frac{\partial F_{n}}{\partial y} Q_{2}=F_{n}\left(a_{01} x-a_{10} y\right) \text {. }
$$

The above equation becomes a particular instance of equation $\left(6 . \mathrm{CT}_{2 ; i, F}\right)$ for $i=n+1$ if we write

$$
\begin{aligned}
& F(x, y)=F_{n+1}(x, y)+F_{n}(x, y)+\cdots+F_{1}(x, y)+F_{0} \\
& \begin{aligned}
\text { with } F_{n+1}(x, y)=a_{n+1,0} x^{n+1}+\cdots+a_{0, n+1} y^{n+1} & \\
& \quad \text { with } a_{i j}=0, i+j=n+1 .
\end{aligned}
\end{aligned}
$$

In general, if we look for an algebraic integral $F(x, y)=0$ of degree $n+1$ after we discussed the algebraic integrals $F(x, y)=0$ of degree $n$, we note that the equations obtained by identifying the terms of degrees $i$ with $i \leq n$ in $\left(6 . \mathrm{CT}_{2}\right)$ are the same for curves of degrees $n$ and $n+1$. The last equation used for curves of degree $n$ is $\left(6 . \mathrm{CT}_{2 ; i, F}\right)$ with $i=n+1$ and $F_{n+1}(x, y)$ with zero coefficients while for a curve of degree $n+1$, we have two other equations (6.CT $\mathrm{T}_{2 ; i, F}$ ) obtained by identifying the remaining coefficients of the terms of the degrees $i=n+2$ and $i=n+1$ in $\left(6 . \mathrm{CT}_{2}\right)$ where we write $F=\sum_{i=0}^{i=n+2} F_{i}$ with $F_{n+2}(x, y)=\sum_{i+j=n+2} a_{i j} x^{i} y^{j}$ with $a_{i j}=0$.

In particular if we want to compute the conics and cubics, we first identify the coefficients of the degree 2 terms and this will give us some of the coefficients of $F_{2}$. These coefficients are also used when we compute the cubic integrals.

We illustrate the computation of invariant algebraic curves with some results concerning the existence of invariant conic curves for a system (3.S'). Straightforward calculations (cf. [34]) yield

Proposition 6.2. Assume that the real system $\left(3 . \mathrm{S}^{\prime}\right)$ has a conic curve $F(x, y)=$ 0 , with real coefficients, passing through the origin and such that $F$ divides $(\partial F / \partial x) P+(\partial F / \partial y) Q$, where $P$ and $Q$ are the polynomials on the right hand side of $\left(3 . \mathrm{S}^{\prime}\right)$. Then the conic is $x^{2}+y^{2}=0$ and the coefficients of the system satisfy the conditions: $C=2 a$ and $A+b-d=0$.

From the identity $\left(6 . \mathrm{CT}_{2}\right)$, straightforward calculations also yield the following:

Proposition 6.3. If a real quadratic system (3.S') satisfying condition (III) has a conic curve $F(x, y)=0$, not passing through the origin as an algebraic particular integral, then this conic is either reducible over $\mathbb{C}$ or else the system can be brought via a coordinate change, to one of the same form $\left(3 . S^{\prime}\right)$, satisfying $b+$ $d=0$ and with $a=0=C=A+2 b$, in which case the conic is

$$
a_{20}\left(x^{2}+y^{2}\right)-2 b y+1=0 \text {. }
$$

Theorem 6.1. Assume that a system (3.S') with $b+d \neq 0$, has a conic curve $F(x, y)=0$, not passing through the origin as an algebraic particular integral. If this conic is irreducible, then we have the following possible situations: 
(i) If $a \neq 0$, then necessarily $d \neq 0$, the coefficients of the system must satisfy the following two equations:

$$
\begin{gathered}
2 d^{2}+b d-a C-a^{2}=0, \\
d(C+a)(A-d)+2 a(C+a)^{2}-a d(2 d+b)=0,
\end{gathered}
$$

and the conic is a parabola whose equation is

$$
\left(d^{2}-a C-a^{2}\right)\left\{(a x-d y)^{2}+2 d y\right\}+d^{2}=0 .
$$

(ii) If $a=0$, then necessarily $C=0$ and hence (II) holds. We have: If $A+b-d \neq 0$, then $b(A+b)(A+2 b) \neq 0$, the conic is unique and its equation in this case is

(6.8) $b(A+b)(A+2 b) x^{2}+b d(A+2 b) y^{2}+2 b y(A+b-d)-(A+b-d)=0$.

If $A+b-d=0$ then $b d=0$ and the conic is anyone of the one-parameter family of curves

$$
a_{20} x^{2}+\left(b^{2}+a_{20}\right) y^{2}-2 b y+1=0 .
$$

Proof. We may assume the conic to be $F(x, y)=0$ where

$$
F(x, y)=a_{20} x^{2}+2 a_{11} x y+a_{02} y^{2}+a_{10} x+a_{01} y+1
$$

with $\left(a_{20}, a_{11}, a_{02}\right) \neq 0$. (6.CT 2$)$ yields $w=0$ and identifying the coefficients of the terms of order one in $\left(6 . \mathrm{CT}_{2}\right)$ we have

$$
u=a_{01} \text { and } v=-a_{10} .
$$

Identifying the coefficients of $x^{2}$ and $y^{2}$ in $\left(6 . \mathrm{CT}_{2}\right)$ we obtain the equations:

$$
\begin{array}{cc}
\text { a. } a_{01}+2 a_{11}-b a_{10}-a_{10} a_{01}=0 & \left(\text { for } x^{2}\right), \\
-a . a_{01}-2 a_{11}-d a_{10}+a_{10} a_{01}=0 & \left(\text { for } y^{2}\right) .
\end{array}
$$

From these we obtain the equation

$$
a_{10}(b+d)=0
$$

and, since $b+d \neq 0$, we get $a_{10}=0$. Replacing this in (6.12) we obtain the equation:

$$
a_{11}=-a \cdot a_{01} / 2 .
$$

Identifying the coefficients of $x y$ in $\left(6 . \mathrm{CT}_{2}\right)$ we obtain

$$
2 a_{02}-2 a_{20}+a_{01} A=a_{01}^{2} \text {. }
$$

From (6.16) we obtain

$$
a_{20}=\left(a_{01} A+2 a_{02}-a_{01}^{2}\right) / 2 .
$$

It remains to identify the coefficients of the terms of degree 3 in $\left(6 . \mathrm{CT}_{2}\right)$, i.e.

$$
\frac{\partial F_{2}}{\partial x} P_{2}+\frac{\partial F_{2}}{\partial y} Q_{2}-F_{2} a_{01} x=0 .
$$

In (6.18) we replace the coefficients given by the formulas (6.15) and (6.17) and identifying the coefficients of the degree 3 terms in (6.18) we obtain the equations:

$$
a\left(a_{01} d-2 a_{02}\right)=0 \quad\left(\text { for } y^{3}\right),
$$




$$
\begin{array}{r}
-a_{01} A b-2 a_{02} b+a_{01}^{2} b-a_{01}^{2} A / 2-a_{01} a_{02}+a_{01}^{3} / 2-a^{2} a_{01}=0, \\
\left(\text { for } x^{3}\right), \\
-a_{01} A d-2 a_{02} d+a_{01}^{2} d+a a_{01} C+2 a_{02} A-a_{01} a_{02}+a^{2} a_{01}=0 \\
\left(\text { for } x y^{2}\right), \\
-a_{01} A C-2 a_{02} C+a_{01}^{2} C+a a_{01} b-a a_{01} A+2 a a_{02}+a a_{01}^{2}=0 \\
\left(\text { for } x^{2} y\right) .
\end{array}
$$
have

$$
a_{01}^{2}-2 a_{02}-a_{01} A=0 .
$$

From this we get

$$
a_{02}=\left(a_{01}^{2}-a_{01} A\right) / 2 .
$$

We are left with two equations: (6.20) and (6.21) where we replace $a_{02}$ from (6.24) and $a$ by zero. (6.20) becomes $0=0$ and after factoring, (6.21) becomes

$$
a_{01}\left(a_{01}-A\right)\left(2 A-a_{01}\right)=0 .
$$

If $a_{01}=0$ then $a_{i j}=0$. The case $a_{01}=A$ yields $a_{i j}=0$ for $i+j=2$. In the case $a_{01}=2 A$ the conic is the double line $(1+A y)^{2}=0$. So $C=0$. Since $a=0$, by (6.15) we have $a_{11}=0$ and identifying with zero the coefficients of $x^{3}$ in (6.18) we have

$$
a_{20}\left(a_{01}+2 b\right)=0 .
$$

If $a_{20}=0$, the conic is $(A y+1)^{2}=0$, so from (6.26) have $a_{01}=-2 b$ and equation (6.16) becomes

$$
-a_{20}+a_{02}=b(A+2 b)
$$

while equation (6.21) after simplification by 2 becomes

$$
(A+b-d) a_{02}+b d(A+2 b)=0 .
$$

If $A+b-d \neq 0$, the linear system $\{(6.26),(6.27)\}$ yields

$$
a_{02}=\frac{-b d(A+2 b)}{A+b-d}, \quad a_{20}=\frac{-b(A+b)(A+2 b)}{A+b-d} .
$$

The conic is

$$
\frac{-b(A+2 b)(A+b) x^{2}}{A+b-d}-\frac{b d(A+2 b) y^{2}}{A+b-d}-2 b y+1=0
$$

and multiplying by $A+b-d$ we obtain (6.8). We observe that this is a true conic if and only if $b(A+2 b) \neq 0$. If $A+b=0$, then (6.30) is a double line. So $A+b \neq 0$. If $A+b-d=0$ then (6.28) yields $b d(A+2 b)=0$. But $A+2 b=d+b \neq 0$. Hence we must have $b d=0$. If $b=0$ then $a_{01}=0=A-d$ and from (6.16) we have $a_{20}=a_{02}$. In this case the conic is

$$
a_{20}\left(x^{2}+y^{2}\right)+1=0 \text {. }
$$


If $d=0$, then $A+b=0$ and (6.27) yields $a_{02}=b^{2}+a_{20}$. In this case we have the one-parameter family of conics (6.9). We note that $(6.31)$ is $(6.9)$ with $b=0$.

Case $a \neq 0$. From (6.19) we get

$$
a_{02}=a_{01} d / 2 \text {. }
$$

Replacing the above in (6.21), we obtain on the left hand side an expression which factors through $a_{01}$. We must have $a_{01} \neq 0$ since otherwise all $a_{i j}$ are zero. So simplifying by $a_{01}$ and eliminating the denominators we obtain

$$
2 d^{2}-a_{01} d-2 a(C+a)=0 \text {. }
$$

Using (6.32) and the above equation, we obtain

$$
a_{02}=d^{2}-a(C+a) \text {. }
$$

We show that we must have $d \neq 0$. Indeed, if we suppose $d=0$, then by (6.32) we must have $a_{02}=0$ and from (6.33) we obtain $C+a=0$. Equation (6.22) yields $a b=0$, hence $b=0$, contradicting our hypothesis that $b+d \neq 0$. So $d \neq 0$ and (6.33) yields

$$
a_{01}=2\left(d^{2}-a(C+a)\right) / d .
$$

Replacing (6.32) in (6.22), after simplifying by $a_{01}$ and replacing in the resulting equation $a_{01}$ from (6.35), we obtain

$$
C d^{2}+3 a d^{2}-A C d+a b d-a A d-2 a C^{2}-4 a^{2} C-2 a^{3}=0 .
$$

Regrouping the terms above and isolating $A$ we obtain

$$
A d(C+a)=(C+a) d^{2}+a d(2 d+b)-2 a(C+a)^{2}
$$

from which we obtain the equation (6.6) which we had to prove. We distinguish two possibilities: $C+a \neq 0$ and $C+a=0$. If $C+a \neq 0$ then we have

$$
A=\left(C d^{2}+3 a d^{2}+a b d-2 a C^{2}-4 a^{2} C-2 a^{3}\right) / d(C+a) .
$$

Replacing (6.32) in (6.20), simplifying by $a_{01}$ and eliminating the denominator, we obtain

$$
2 b d+a_{01} d+2 A b-2 a_{01} b+a_{01} A-a_{01}^{2}+2 a^{2}=0 .
$$

Replacing $(6.35)$ in the above, eliminating the denominator and simplifying by 2 , we get

$$
\begin{aligned}
d^{4}+b d^{3}-A d^{3}-3 a C d^{2}-A b d^{2}-4 a^{2} d^{2}-2 a b C d+a A C d & \\
& -2 a^{2} b d+a^{2} A d+2 a^{2} C^{2}+4 a^{3} C+2 a^{4}=0 .
\end{aligned}
$$

Using (6.38) in the above and factoring we obtain

$$
-a d^{2}(b+d)\left(2 d^{2}+b d-a C-a^{2}\right)=0,
$$

and since $a d^{2}(b+d) \neq 0$, we obtain the equation (6.5) we had to prove. From (6.5) we get

$$
b=-\left(2 d^{2}-a C-a^{2}\right) / d .
$$

Using the above together with (6.34), (6.35), and (6.38) in (6.17) and (6.15) we obtain the equation (6.7) for the conic. 
Assume now that $a d \neq 0=C+a$. In this case (6.37) yields $2 d+b=0$. The formula (6.33) yields $a_{01}=2 d$ and (6.34) yields $a_{02}=d^{2}$. Using these and (6.17) we get $a_{20}=d(A-d)$. Using formula (6.15) we get $a_{11}=-a d$. From these, (6.20) yields $-A b+b d-A d+d^{2}-a^{2}=0$ and after replacing $b$ by $-2 d$ we get $-d^{2}+A d-a^{2}=0$ or $a^{2}=d(A-d)$. Hence the equation of the conic is $a^{2} x^{2}-2 a d x y+d^{2} y^{2}+2 d y+1=0$ or $(a x-d y)^{2}+2 d y+1=0$ which is (6.7) with $a+C=0 \neq a d$.

Remark 6.2. We note that if in the above theorem both the system and the curve are taken with real coefficients, then in view of the Proposition 6.1, the assumption that the curve pass through the origin may be dropped. In this case, if $a=0$ the origin is a center and the system is integrable.

Corollary 6.1. Assume that a quadratic system has a weak focus of order 1. Suppose that this system has an invariant conic curve, irreducible over $\mathbb{C}$. Then this conic is a parabola.

We observe that for the case of a degenerate focus, from the Proposition 6.3 and the Theorem 6.1 and the calculations used in the proof of Theorem 6.1, we obtain the following result which connects the existence of an invariant parabola with the equation $a^{2}+2 d^{2}+b d=0$ in condition (I) for the center.

Corollary 6.2. Consider a real system (3.S') which has a degenerate weak focus at the origin. Assume that the system has a conic curve $F(x, y)=0, F(x, y) \in$ $\mathbb{R}[x, y]$, not passing through the origin, which is an algebraic particular integral for the system. Then the following conditions hold:

(i) The coefficients of the system must satisfy the equation

$$
a(b+d)\left(2 d^{2}+a^{2}+b d\right)=0 .
$$

(i) The origin is a center and the system is integrable.

Furthermore, if the conic is a parabola, then $b+d \neq 0$ and if $a \neq 0$, we also have $d \neq 0$ and

$$
A+3 b+5 d=0=2 d^{2}+a^{2}+b d .
$$

Corollary 6.3. A real quadratic system with a weak focus of order 2 or 3 has no real algebraic integral which is an irreducible conic curve.

Corollary 6.4. $A$ real quadratic system with a weak focus $F$ and with an irreducible conic curve as an algebraic integral has either a center or a weak focus of order 1 at $F$.

The following theorem connects the condition $A+3 b+5 d=0$ in (I) with the existence of a particular cubic integral.

Theorem 6.2. Assume that a system (3.S') with real coefficients has a degenerate weak focus at the origin. Let us suppose that this system has a cubic curve $F(x, y)=0$ with real coefficients, not passing through the origin, which is an algebraic particular integral of the system. Then:

(i) The coefficients of the system necessarily satisfy the equation

$$
a(b+d)(A-2 b)(A+3 b+5 d)=0
$$

and if $a(b+d)(A-2 b) \neq 0$, then the system satisfies condition (I). 
(ii) The origin is a center. [36]).

The proof of this theorem is analogous to the proof of Theorem 6.1 (cf.

\section{A GENERIC CHARACTERIZATION OF QUADRATIC SYSTEMS WITH A CENTER}

In this section we determine the geometric content of the conditions for the center denoted by (III), (II), (I). These conditions correspond respectively to the three geometric properties denoted by $\mathbf{P}_{\mathrm{III}}, \mathbf{P}_{\mathrm{II}}, \mathbf{P}_{\mathrm{I}}$ in $\S 1$. We show two things: (1) that if a system satisfies the property $\mathbf{P}_{i}$ then, via a coordinate change we can bring the system to the form $\left(3 . S^{\prime}\right)$ and such that its coefficients satisfy the condition $i, i=\mathrm{III}, \mathrm{II}, \mathrm{I}$; and (2) that the property $\mathbf{P}_{i}$ is generic in the set $\mathbf{C}_{i}$ with respect to the usual topology. Each property $\mathbf{P}_{i}$ says that the system possesses two low degree algebraic particular integrals of specific types. In $\S 6$ we obtained the conditions on the coefficients of a quadratic system with a weak focus, in case the system possesses one algebraic integral of low degree. Now we consider the case of two algebraic particular integrals and the conditions we obtain are those for the center.

Let us first assume that part (1) has been proved and let us show part (2). We consider first the cases $i=$ III, II. In these cases, with the possible exception of bifurcation points, the system has an invariant straight line $L$ and a conic particular integral which is not $L$ with multiplicity 2 , both not passing through the origin. Indeed as seen in $\S 4$, for the case (III), we may assume $a=0$ and in this case the conic integral (reducible over $\mathbb{C}$ ) is

$$
(C x / 2-b y+1)^{2}-\Delta x^{2} / 4=0
$$

and in case (II) with $A b(A+b)(A+2 b) \neq 0$, the conic integral is (6.8). We turn now to condition (I) for the center. We see that if a system $\left(3 . \mathrm{S}^{\prime}\right)$ satisfies (I) and $a \neq 0$, then $d \neq 0$ and the system has the following parabola as a particular integral

$$
d^{2}+2 d\left(d^{2}+a^{2}\right) y+\left(d^{2}+a^{2}\right)(a x-d y)^{2}=0 .
$$

Observing the first integral for $(\mathrm{I})$, we see that the following cubic is a particular integral

(7.3)

$d^{2}+3 d\left(d^{2}+a^{2}\right) y+3 d^{2}\left(d^{2}+a^{2}\right) y^{2}-3 a d\left(d^{2}+a^{2}\right) x y-\left(d^{2}+a^{2}\right)(a x-d y)^{3}=0$.

We note that this cubic has no singular point in the affine plane and that it has a singular point at infinity which coincides with the one of the parabola. If this cubic were reducible, then this point would belong to the components. Since the curve has no terms in $x$ or in $x^{2}$, this implies that the curve is irreducible. Thus a system satisfying (I) with $a \neq 0$, also satisfies $\mathbf{P}_{I}$ and hence $\mathbf{P}_{I}$ is a generic property in $\mathbf{C}_{\mathrm{I}}$.

It remains to prove the part denoted by (1) above. We first consider conditions (III) and (II). It follows from the Proposition 4.1, that if a system $\left(3 . \mathrm{S}^{\prime}\right)$ has two distinct straight lines (possibly with complex coefficients) as integrals, then the system satisfies (III). We also have the following:

Theorem 7.1. Assume that a system (3.S') with real coefficients possesses an invariant straight line $L$ and a conic integral $F(x, y)=0, F(x, y) \in \mathbb{R}[x, y]$, 
which is not the line $L$ with multiplicity 2 . Then the system satisfies one of the two conditions (III): $b+d=0$ or (II): $a=0=C$. If the conic integral is irreducible then in some coordinate axes, the system satisfies (II): $a=0=C$.

Proof. By Proposition 4.1, $b+d=0$, or $a=0 \neq A$; assume that $b+d \neq 0$, then $a=0 \neq A$, the system is of the form

$$
\frac{d x}{d t}=-y-b x^{2}-C x y-d y^{2}, \quad \frac{d y}{d t}=x+A x y
$$

and $1+A y=0$ is the invariant straight line. If the conic passes through the origin, then in view of Proposition 6.2 we must have $C=2 a$ and since $a=0$, $C=0$. If the conic does not pass through the origin we may assume it to be $F(x, y)=0$ with $F$ given by (6.10). The calculations in the initial part of the proof of Theorem 6.1, using (6.CT $)$, apply here and we have (6.12), (6.13) and hence (6.14) from which $a_{10}=0$. From (6.12) we get $a_{11}=0$. Identifying the coefficients of $x^{2} y$ in $\left(6 . \mathrm{CT}_{2 ; n+1, F}\right)$ with $n=2$ we obtain $a_{20} C=0$. If we assume $a_{20}=0$, then since $a_{11}=0$ we must have $a_{02} \neq 0$. Identifying the coefficients of $x y^{2}$ in $\left(6 . \mathrm{CT}_{2 ; n+1, F}\right)$ with $n=2$, we obtain the equation

$$
a_{02}\left(2 A-a_{01}\right)=0
$$

hence $a_{01}=2 A$. Identifying the $x y$ coefficients in $\left(6 . \mathrm{CT}_{2 ; n+1, F}\right)$ with $n=2$ and using the other coefficients we obtained so far yields us $a_{02}=A^{2}$ and hence the conic is $(1+A y)^{2}=0$, a case ruled out by our hypothesis. So necessarily we have $a_{20} \neq 0$ and $C=0$. The last part of the theorem is obtained using Proposition 6.3.

We now turn to condition (I).

Theorem 7.2. Assume that a system (3.S') with real coefficients has a parabola and an irreducible cubic curve as algebraic particular integrals. Assume that the cubic integral has only one point at infinity which coincides with the one of the parabola and that it does not pass through the origin. Then the origin is a center, the coefficients of the system satisfy the conditions

$$
C+2 a=A+3 b+5 d=a^{2}+2 d^{2}+b d=0
$$

and the cubic curve has equation (7.3).

Proof. By Propositions 6.1, 6.3, and Theorem 6.1 we must consider two possibilities. First we consider the case $a=0$ in which case $C=0$ and the origin is a center. From Theorem 6.1 we see that the only situation when we could have a parabola is the case when $d=0$. In this case the equation for the parabola is (6.8). The point at infinity of the homogenized equation is $x=0, y=1$, $z=0$. By hypothesis, this is also the point at infinity of the cubic integral, so the only cubic term of the cubic integral is $B x^{3}$ with $B \neq 0$. Using the observation in $\S 6$ regarding the calculation of the cubic integrals after we have computed the conic ones, from (6.12), (6.13) with $a=0$ and from (6.14) we obtain that there are no terms in $x$ or in $x y$ in the equation of the cubic. So the cubic must be of the form:

$$
B x^{3}+a_{20} x^{2}+a_{02} y^{2}+a_{01} y+1=0 .
$$

Identifying the coefficients of $x^{2} y$ in equation $\left(6 . \mathrm{CT}_{2 ; i, F}\right)$ for $i=3$ we obtain $B=0$ which is a contradiction. So we must have $a \neq 0$ in which case $d \neq 0$ 
and by Theorem 6.1 the parabola has equation (6.7) and the coefficients of the system satisfy conditions (6.5) and (6.6). Since $a \neq 0$, from equation (6.5) we obtain

$$
C=\left(2 d^{2}+b d-a^{2}\right) / a .
$$

Introducing this in (6.6), eliminating the denominator $a$ and then factoring we obtain

$$
d(2 d+b)\left(3 d^{2}+2 b d+A d-a^{2}\right)=0 .
$$

Since $d \neq 0$, we have two possibilities: $b=-2 d$ and

$$
3 d^{2}+2 b d+A d-a^{2}=0 .
$$

Suppose first that $b=-2 d$. In this case (7.7) yields $C=-a$. We may assume the cubic curve is of the from $F=F_{3}+F_{2}+F_{1}+1=0$ with $F_{i}$ homogeneous of degree $i$. Since the cubic has only one point at infinity, coinciding with the one of the parabola (6.7), we must have $F_{3}(x, y)=B(a x-d y)^{3}$ with $B \neq 0$. The calculations at the beginning of the proof of Theorem 6.1 apply for our cubic $F$ and (6.12), (6.13) hold and hence also (6.14). Since the system has a parabola as integral, $b+d \neq 0$ and so $a_{10}=0$ and hence we also have (6.15). The coefficient $a_{20}$ is given by the formula (6.17). Identifying the terms of degree 3 in $\left(6 . \mathrm{CT}_{2}\right)$, we have (using $\left.a_{01}=u\right)$ :

$$
\frac{\partial F_{3}}{\partial x} P_{1}+\frac{\partial F_{3}}{\partial y} Q_{1}+\frac{\partial F_{2}}{\partial x} P_{2}+\frac{\partial F_{2}}{\partial y} Q_{2}-F_{2}(x, y) u x=0
$$

where $P_{i}, Q_{i}$ denote the terms of degree $i$ in the right-hand side of equations $\left(3 . S^{\prime}\right)$. Identifying the terms in $y^{3}$ we obtain

$$
-a\left(2 a_{02}-d u+3 B d^{2}\right)=0 .
$$

Simplifying by $a$ and solving for $a_{02}$ we have

$$
a_{02}=d(u-3 B d) / 2 \text {. }
$$

Next, we equate with zero the coefficient of $x^{2} y$ in (7.10). After replacing in the equation thus obtained the value for $a_{02}$ given by (7.12), and using $C=-a$, after factoring we obtain

$$
a\left(2 d u+b u-3 a^{2} B\right)=0 .
$$

If $a \neq 0$, under the assumption $2 d+b=0$, from the above equation we obtain $B=0$. So we must have $2 d+b \neq 0$. This together with $d \neq 0$ and (6.5), yield $C+a \neq 0$ and equation (7.9) holds. From (7.9) we obtain

$$
A=\left(a^{2}-3 d^{2}-2 b d\right) / d .
$$

We replace the above value of $A$ in $Q_{2}(x, y)$ and we identify the terms of degree 4 in $\left(6 . \mathrm{CT}_{2}\right)$. These give us the equation

$$
\frac{\partial F_{3}}{\partial x} P_{2}+\frac{\partial F_{3}}{\partial y} Q_{2}-F_{3} u x=0 .
$$

Identifying the coefficients of $x y^{3}$ above, dividing by $d^{3}$ and solving for $u$, we obtain

$$
u=-3 b-3 d
$$


The coefficients of the other terms of degree 4 in (7.15) are respectively

$$
\begin{array}{cl}
-3 a d^{2}(u+3 b+3 d) & \left(\text { for } x^{2} y^{2}\right), \\
3 a^{2} d(u+3 b+3 d) & \left(\text { for } x^{3} y\right) \\
-a^{3}(u+3 b+3 d) & \left(\text { for } x^{4}\right)
\end{array}
$$

All these coefficients become zero if and only if (7.16) holds. Replacing the value of $u$ from (7.16) in (7.12) we obtain

$$
a_{02}=-3 d(B d+d+b) / 2 .
$$

Replacing the above in the expression of the coefficient of $x y^{2}$ in (7.10), after factoring and multiplying by $2 / 3 d$ we obtain the equation

$$
\left(3 d^{2}+b d+2 a^{2}\right) B+(b+d)^{2}=0 .
$$

Consider now the coefficient of $x^{2} y$ in (7.10). After replacing the value of $a_{02}$ given by (7.20), factoring and then multiplying by $a / 3$ we obtain the equation

$$
\left(2 d^{4}+b d^{3}-a^{4}\right) B+d(2 d+b)(b+d)^{2}=0 .
$$

Consider now the coefficient of $x^{3}$ in (7.10). After replacing the value of $a_{02}$ like before, factoring and multiplying by $-2 d / 3$ and then regrouping the terms we obtain the equation

$$
d^{2}\left(3 d^{2}+b d+2 a^{2}\right) B+(b+d)^{2}\left(3 d^{2}+b d+a^{2}\right)=0 .
$$

We need to have a joint solution for equations (7.21), (7.22), and (7.23). We isolate $(b+d)^{2}$ from equation (7.21) and replace it in (7.22) and (7.23); after factoring, we obtain respectively

$$
\begin{gathered}
\left(a^{2}+2 d^{2}+b d\right)^{2}=0, \\
-\left(3 d^{2}+b d+2 a^{2}\right)\left(2 d^{2}+b d+a^{2}\right) B=0 .
\end{gathered}
$$

From (7.24) we have

$$
a^{2}+2 d^{2}+b d=0 .
$$

Isolating $a^{2}$ from (7.26) and replacing this in (7.14) and in (7.7) respectively, we obtain

$$
\begin{gathered}
A+3 b+5 d=0, \\
C+2 a=0 .
\end{gathered}
$$

(7.26), (7.27), and (7.28) are conditions (I) and hence by Theorem 3.1 we have a center at the origin. Let us now calculate the coefficients of the cubic. Using (7.26) in (7.21) we obtain the following expression for $B$ :

$$
B=-(b+d)^{2} /\left(a^{2}+d^{2}\right) .
$$

We calculate the remaining coefficients of the cubic. First we solve (7.26) for $b$ and obtain

$$
b=-\left(2 d^{2}+a^{2}\right) / d .
$$


Replacing the above in (7.29) and in (7.16) we obtain respectively

$$
\begin{gathered}
B=-\left(d^{2}+a^{2}\right) / d^{2}, \\
u=3\left(d^{2}+a^{2}\right) / d .
\end{gathered}
$$

We replace the above in (6.15) and we obtain

$$
a_{11}=-3 a\left(d^{2}+a^{2}\right) / 2 d .
$$

We replace (7.30) and (7.31) in (7.20) and we obtain

$$
a_{02}=3\left(d^{2}+a^{2}\right) \text {. }
$$

Replacing the above and (7.32) in (6.16) yields

$$
a_{20}=3 d(b+d)\left(2 d^{2}+b d+a^{2}\right) / 2\left(d^{2}+a^{2}\right)
$$

which in view of (7.26) yields $a_{20}=0$. Hence the invariant cubic is

$$
-\frac{\left(d^{2}+a^{2}\right)(a x-d y)^{3}}{d^{2}}-\frac{3 a\left(d^{2}+a^{2}\right) x y}{d}+3\left(d^{2}+a^{2}\right) y^{2}+\frac{3\left(d^{2}+a^{2}\right) y}{d}+1=0
$$

and eliminating the denominators we obtain (7.3).

Since the degenerate singularities of quadratic vector fields cannot be centers (cf. $[4,43,17]$ ), the results in this paragraph give us the generic geometric characterization of the center in terms of the algebraic integrals of degrees less than or equal to 3 . If we want to use real curves, the generic geometric characterization can be stated as follows:

Theorem 7.3. The following property $\mathbf{P}$ is generic for the real plane quadratic systems with a center: The system has a weak focus $F$ and it is either Hamiltonian or it has a parabola as particular integral and a real irreducible cubic integral, not passing through $F$ and which has only one point at infinity coinciding with the one of the parabola, or it has a real invariant straight line and an irreducible real conic integral, or it has as algebraic particular integrals a real straight line $L$ and a real conic curve, reducible over $\mathbb{C}$, which is not $L$ counted with multiplicity 2 and which does not pass through $F$. Furthermore, if a quadratic system has the property $\mathbf{P}$ then the system has a center at $F$.

\section{Algebraic PARTICULAR INTEgRALS AND INTEGRABILITY, DARBOUX'S THEOREM}

In this paragraph we consider the results obtained in this work in the perspective of Darboux's work [7] in which he gives the general integral of a differential equation defined by polynomials, if we know sufficiently many irreducible algebraic particular integrals of the equation.

In [7] Darboux considers differential equations over the projective plane of the form

$$
L(y d z-z d y)+M(z d x-x d z)+N(x d y-y d x)=0
$$

where $L, M, N$ are homogeneous polynomials in the homogeneous coordinates $x, y, z$. 
Darboux considered particular solutions of (8.1) which are algebraic curves

$$
f(x, y, z)=0
$$

with $f$ a homogeneous polynomial, i.e., curves (8.2) such that the form $d f$ is zero at a point on the curve, on the integral curves of the equation, i.e., such that on $(8.2)$ we have

$$
\frac{\partial f}{\partial x} d x+\frac{\partial f}{\partial y} d y+\frac{\partial f}{\partial z} d z=0
$$

in the direction of the integral curves. Since $f$ is homogeneous, we also have

$$
x \frac{\partial f}{\partial x}+y \frac{\partial f}{\partial y}+z \frac{\partial f}{\partial z}=0 .
$$

From the last two equations, we deduce:

$$
\frac{\partial f / \partial x}{y d z-z d y}=\frac{\partial f / \partial y}{z d x-x d z}=\frac{\partial f / \partial z}{x d y-y d x}
$$

and hence using (8.1) we obtain

$$
L \frac{\partial f}{\partial x}+M \frac{\partial f}{\partial y}+N \frac{\partial f}{\partial x}=0 .
$$

Darboux remarks that this equation is not necessarily an identity. In fact equation (8.6) takes place on the curve (8.2). Darboux says: "It will suffice that the preceding equation takes place only on the points of the curve i.e. that the first member be divisible by $f$ and that we have identically:

$$
L \frac{\partial f}{\partial x}+M \frac{\partial f}{\partial y}+N \frac{\partial f}{\partial z}=K f
$$

$K$ denoting a polynomial which would necessarily be of degree $m-1$, one less than the degree of that of $L, M, N$ ".

Remark 8.1. Darboux's work [7], published in 1878, appeared before Hilbert's nullstellensatz. Darboux replaces there " $(8.6)$ holds on all points of the curve (8.2)" with (8.7). This of course holds for irreducible curves (8.2), when we work over the complex numbers. Darboux calls such a particular integral, a particular algebraic solution of the equation.

We are interested here in polynomial vector fields or in first order equations on the affine plane, not necessarily homogeneous. When written in homogeneous coordinates, a differential equation

$$
d y / d x=f(x, y) / f_{1}(x, y)
$$

with $f, f_{1}$ polynomials, is a particular case of $(8.1)$. Indeed, written in homogeneous coordinates, after replacing $x, y$ by $x / z, y / z$ respectively, this equation gives

$$
\phi(x, y, z)(z d x-x d z)+\phi_{1}(x, y, z)(y d z-z d y)=0
$$

with $\phi, \phi_{1}$ homogeneous polynomials of the same order. The above equation is a particular instance of $(8.1)$, i.e. the case when $N=0$. This equation admits the particular solution $z=0$, i.e., the straight line at infinity. 
In the first part ${ }^{1}$ of Darboux's work [7], he states a theorem saying that if we have sufficiently many algebraic particular integrals of equation (8.1) then we have a first integral of the equation.

We consider here a version of Darboux's theorem stated in the first part of [7, p. 79] which applies to this particular situation.

Theorem 8 (Darboux). Let us consider a vector field

$$
X(x, y)=P(x, y) \partial / \partial x+Q(x, y) \partial / \partial y
$$

with $P, Q$ polynomials over $\mathbb{C}$ with $m=\max (\operatorname{deg} P, \operatorname{deg} Q)$. Let us suppose that this vector field admits $q$ algebraic particular integrals $f_{i}=0$ with $i=$ $1, \ldots, q$, which are irreducible over $\mathbb{C}$. Let $\operatorname{dim}_{\mathbb{C}} \mathbb{C}_{m-1}[x, y]$ be the dimension of the complex vector space $\mathbb{C}_{m-1}[x, y]$ of polynomials of degrees at most $m-1$ in the indeterminates $x$ and $y$ and let us suppose that $q>\operatorname{dim}_{\mathbb{C}} \mathbb{C}_{m-1}[x, y]$. Then the vector field has a first integral of the form

$$
f_{1}^{\lambda_{1}} f_{2}^{\lambda_{2}} \cdots f_{q}^{\lambda_{q}}
$$

Proof. By hypothesis we have irreducible algebraic integrals $f_{i}(x, y)=0$ of $X$, i.e., $f_{i}$ 's are polynomials such that $d f_{i}(X)=K_{i} f_{i}$ with $K \in \mathbb{C}_{m-1}[x, y]$. By hypothesis we have $q$ such integrals with $q>\operatorname{dim}_{\mathbb{C} m-1}[x, y]=1+2+\cdots+m=$ $m(1+m) / 2$ and hence we have $q$ such polynomials $K_{i}$ of degree $m-1$. These $K_{i}$ 's must be linearly dependent, i.e., we have complex $\lambda_{j}$ 's such that $\sum_{j=1}^{q} \lambda_{j} K_{j}=0$. We obtain $\left(\sum_{j=1}^{q} \lambda_{j} d f_{j} / f_{j}\right)(X)=0$, i.e., $f_{1}^{\lambda_{1}} \cdots f_{q}^{\lambda_{q}}$ is a first integral of $X$.

Remark 8.2. We note that we may have algebraic particular integrals $f(x, y)=$ 0 with $f$ a polynomial with real coefficients, which have no point or only a finite number of points in the finite plane and these will produce an integral (8.10) which is a complex multivalued function. For a real constant of motion we apply absolute values, i.e., $\left|f_{1}\right|^{\alpha_{1}}|f|_{2}^{\alpha_{2}} \cdots\left|f_{q}\right|^{\alpha_{q}}$.

If we have $q$ algebraic integrals with $q>m(m+1) / 2+1$ of a vector field of degree $m$, then the exponents $\lambda_{1}, \lambda_{2}, \ldots, \lambda_{q}$ in the constant of motion $f_{1}^{\lambda_{1}} f_{2}^{\lambda_{2}} \cdots f_{q}^{\lambda_{q}}$ can be taken to be integers (cf. [41]). Hence in the case given by Darboux's theorem we have a rational constant of motion. In the case not covered by Darboux's theorem, i.e., the case when $q<m(m+1) / 2$, we may still have $q$ linearly dependent $K_{i}$ 's and so we may still get a constant of motion. This is the situation we encountered for systems with a center in the quadratic case. To apply Darboux's theorem in the particular case of quadratic vector fields, i.e., $m=2$, we need $q$ algebraic irreducible integrals, with $q$ greater than 3. From four such particular integrals, Darboux's theorem gives us a first integral of the system which will be rational. Our work shows that for a system with a weak focus, for each one of the generic cases (III), (II), (I), two such algebraic integrals, not passing through the origin, suffice for a constant of motion: either a straight line and a conic or a conic and a cubic curve. In particular we point out that we have not used the first integrals of quadratic systems with a center in the proof of Theorem 7.2 and thus the calculations performed there give us also a first integral for systems satisfying (I) by using the two algebraic

\footnotetext{
${ }^{1}$ This part was not available to the author at the time when this research was done. The author is thankful to R. Moussu who told her Darboux's theorem and the version we use here.
} 
integrals we obtained from the hypothesis of the theorem. Analogously, we have first integrals via algebraic particular integrals for the generic cases of type (II) and (III) and clearly all these first integrals are elementary.

To compute the algebraic particular integrals $F(x, y)=0$ of degree $n$ for a polynomial vector field $d x / d t=P(x, y), d y / d t=Q(x, y)$ of degree $m$, one uses the polynomial identity (6.CT) of degree $n+m-1$ and without constant terms. This leads to $(n+m-1)(n+m) / 2-1$ equations involving the coefficients $a_{k s}, b_{k s}, f_{k s}, g_{k s}$ of the terms in $x^{k} y^{s}$ in $P, Q, F$, and $K$ respectively. We obtain $(n+m-1)(n+m) / 2-1$ equations in the unknowns $f_{k s}, g_{k s}$ and the parameters $a_{k s}, b_{k s}$ of the system. To compute the general integrals we need enough algebraic particular integrals $F_{j}=0$, i.e., such that the corresponding $K_{j}$ 's are linearly dependent. By eliminating the unknown $F_{k s, j}$, $g_{k s, j}$, one finds the compatibility conditions in terms of the parameters $a_{k s}$, $b_{k s}$. When these hold, solving the polynomial equations in the unknowns $f_{k s, j}$, $g_{k s, j}$ in terms of the parameters gives us the particular algebraic integrals. The coefficients $g_{k s, j}$ of the polynomials $K_{j}$ are needed in order to obtain the coefficients of the linear dependence relation required for the calculation of the first integral.

The calculations of algebraic particular integrals of polynomial systems could be tedious and lengthy even when using computers. Such calculations could be programmed in MACSYMA or REDUCE for example. These are large, selfcontained systems which do not incorporate mathematical objects like rings, ideals, and differential forms. Two programs especially designed for commutative algebra are: MACAULEY [42] and COCOA [11]. While these programs are efficient for commutative algebra purposes, they have drawbacks for the kind of calculations we need here. For example one cannot factor polynomials in MACAULEY or in COCOA. This is a significant drawback here since we obtained the conditions for the center by factoring polynomials. Once the polynomials are factored, one can check the factorization by hand and hence obtain a real proof. Usually, it is not possible to obtain the factorization by hand, the calculations being too massive. In view of these observations, more work is needed in order to provide us with a program which could efficiently handle the problem of finding compatibility conditions in terms of parameters and of solving polynomial equations analogous to those encountered in this work. At this stage it seems that the best canditate for accommodating such a program is MAPLE.

\section{CONCLUDING COMMENT AND OPEN PROBLEMS}

As we have seen in this work, there is an algebraic geometric structure associated to the notion of center, in the case of quadratic differential systems. It is natural to ask whether there is a corresponding structure in the case of the cubic systems. Work on the special case of cubic symmetric systems with a center, done by the author together with $\mathrm{Ch}$. Rousseau, shows that the algebraic integrals play a role in these cases, which in part is analogous to what happens in the quadratic situation. At the conference on bifurcations of planar vector fields held at Luminy in September 1989, where the author reported on this work, $\mathrm{N}$. Lloyd reported connections between algebraic particular integrals and the problem of the center obtained with $\mathrm{C}$. Christopher in two other special cases 
of cubic systems but no geometric generic characterization in terms of algebraic particular integrals was known by them at that time. It is natural to consider the problem: Determine the role of the algebraic integrals in the theory of the center for general cubic systems. What are the generic geometric properties of the cubic systems with a center?

\section{ACKNOWLEDGMENT}

The author is very grateful to $\mathbf{J}$. Guckenheimer for raising her interest in the problem of the center and for asking the key question of the geometrical meaning of the algebraic conditions for the center. The author is also very grateful to J. Hubbard and to A. Douady for taking an interest in this work and to A. Douady, J. Guckenheimer, J. Hubbard, and J. Hurtubise for interesting and stimulating mathematical discussions. The author also thanks R. Roussarie who carefully read the manuscript of [32] and who raised some interesting points concerning that first part of this work. Thanks are also due to R. Moussu who told the author about Darboux's theorem. Joan C. Artés noticed a wrong ending of a separatrix in a phase portrait in [32]. Thanks are due to A. Gasull and to J. Llibre for telling the author about this. The author wishes to thank the referee for his suggestions concerning improvements in the exposition and the referee, Ch. Rousseau and J. Carette for final corrections of the manuscript.

This work was done while the author was partly detached to the Centre de Recherche Mathématique de l'Université de Montréal.

\section{BIBLIOGRAPHY}

1. D. V. Anosov and V. I. Arnold (Editors), Dynamical systems, vol. I, Encyclopaedia of Mathematical Sciences, Springer-Verlag, Berlin and New York, 1988, p. 233.

2. V. I. Arnold, Chapitres supplémentaires de la théorie des équations différentielles ordinaires, "Mir," Moscow, 1980, p. 323. (Translation of the Russian 1978 edition published by Nauka.)

3. N. N. Bautin, On the number of limit cycles which appear with the variation of the coefficients from an equilibrium position of focus or center type, Mat. Sb. 30 (72) (1952), 181-196; English transl. in Math. USSR-Sb. 100 (1954), 397-413.

4. V. Berlinskii, On the behaviour of the integral curves of a differential equation, Izv. Vyssh. Uchebn. Zaved. Mat. 2 (15) (1960), 3-18. (Russian)

5. F. Browder (Editor), Mathematical developments arrising from Hilbert's problems, Proc. Sympos. Pure Math., vol. 28, Amer. Math. Soc., Providence, R.I., 1976, pp. 50-51.

6. Yuanshun Chin (Yuanshun Qin), On surfaces defined by ordinary differential equations, Lecture Notes in Math., vol. 1151, Springer-Verlag, Berlin and New York, 1985, pp. 115131.

7. G. Darboux, Mémoire sur les équations différentielles algébriques du premier ordre et $d u$ premier degré (Mélanges), Bull. Sci. Math. (1878), 60-96, 123-144, 151-200.

8. T. D. Davies and E. M. James, Nonlinear differential equations, Addison-Wesley, Reading, Mass., 1966, p. 191.

9. H. Dulac, Détermination et integration d'une certaine classe d'èquations différentielle ayant pour point singulier un centre, Bull. Sci. Math Sér. (2) 32 (1908), no. 1, 230-252.

10. M. Frommer, Über das Auftreten von Wirbeln und Strudeln (geschlossener und spiraliger Integralkurven) in der Umgebung rationaler Unbestimmheitsstellen, Math. Ann. 109 (1934), 395-424.

11. A. Giovini and G. Niesi, Cocoa user's manual, Department of Mathematics, University of Genova, 1989. 
12. F. Göbber and K. D. Willamowski, Ljapunov approach to multiple Hopf bifurcation, J. Math. Anal. Appl. 71 (1979), 333-350.

13. E. A. González Velasco, Generic properties of polynomial vector fields at infinity, Trans. Amer. Math. Soc. 143 (1969), 201-222.

14. J. Guckenheimer, R. Rand, and D. Schlomiuk, Degenerate homoclinic cycles in perturbations of quadratic hamiltonian systems, Nonlinearity 2 (1989), 405-418.

15. D. Hilbert, Mathematische Probleme (lecture), Second Internat. Congress Math. Paris, 1900, Nachr. Ges. Wiss. Gottingen Math.-Phys. Kl.1900, pp. 253-297; reprinted in [5, p. 134].

16. Ju. S. Il'yashenko, Perturbations of the polynomial Hamiltonian equations in the real and complex domains, Sympos. Methods of the Qualitative Theory and the Theory of Bifurcations, Gorky, 1988, pp. 63-67.

17. P. de. Jager, Phase portraits of quadratic systems-higher order singularities and separatrix cycles, Doctoral Thesis, May 1989, Technische Universiteit Delft.

18. W. Kapteyn, On the midpoints of integral curves of differential equations of the first degree, Nederl. Akad. Wetensch. Verslag. Afd. Natuurk. Konikl. Nederland. (1911), 1446-1457. (Dutch)

19. N New investigations on the midpoints of integrals of differential equations of the first degree, Nederl. Akad. Wetensch. Verslag Afd. Natuurk. 20 (1912), 1354-1365; 21, 27-33. (Dutch)

20. I. S. Kukles and M. Khasanova, O povedenii harakteristik odnovo diferentialnovo uravneniia v kruge Poincare, Dokl. Akad. Nauk Tadzhik SSR 7 (1964), no. 12, 3-6.

21. E. Landis and I. Petrovski, Letter to the editor, Mat. Sb. 73 (1967), 160.

22. Kh. R. Latipov, O Razredelenii osobih tochek uravneniya Frommer na vsei ploskosti, Izv. Vyssh. Uchebn. Zaved. Mat. 1 (44) (1965), 96-104.

23. Kh. R. Latipov and I. I. Shirov, Investigations of differential equations, Izv. Akad. Nauk UzSSR. Sec. Fiz.-Mat. Nauk (1963), 117-131. (Russian)

24. Y. C. Lu, Singularity theory and an introduction to catastrophe theory. Springer-Verlag, Berlin and New York, 1976, p. 199.

25. N. A. Lukashevich, Integral curves of a certain differential equation, Differentsial'nye Uravneniya 1 (1965), no. 1, 82-95.

26. V. A. Lunkevich and K. S. Sibirskii, Integrals of a general quadratic differential system in cases of a center, Differential Equations 18 (1982), no. 5, 786-792.

27. M. A. Lyapunov, Problème général de la stabilité de mouvement, Princeton Univ. Press, Princeton, N.J., 1947, pp. 1-474. (First printing 1892, Harkov)

28. I. Petrovski and E. Landis, On the number of limit cycles of the equation $d x / d y=P(x, y) /$ $Q(x, y)$ where $P$ and $Q$ are polynomials of the second degree, Mat. Sb. 37 (1955), 209250; Amer. Math. Soc. Transl. (2) 10 (1958), 177-221.

29. H. Poincaré, Mémoire sur les courbes définies par les équations différentielles, J. de Math. (3) 37 (1881), 375-422; 8 (1882), 251-296; Oeuvres de Henri Poincaré, vol. I, Gauthier-Villars, Paris, 1951, pp. 3-84.

30. __ Mémoire sur les courbes définies par les équations différentielles, J. Math. Pures Appl. (4) 1 (1885), 167-244; Oeuvres de Henri Poincaré, vol. I, Gauthier-Villars, 1951, Paris, pp. 95-114.

31. L. S. Pontrjagin, Über Autoschwingungssysteme, die den hamiltonschen nahe liegen, Physikalische Zeitschrift der Sowjetunion, Band 6, Heft 1-2, 1934, pp. 25-28.

32. D. Schlomiuk, The "center-space" of plane quadratic systems and its bifurcation diagram, Rapport de Recherche du Département de Mathématiques et de Statistique, D.M.S. No 88-18, L'Université de Montréal, 1988.

33. __ Invariant reducible cubics and the conditions for the center, Rapport de Recherche du Département de Mathématiques et de Statistique, D.M.S. No 89-11, 1989.

34. __ Invariant conics of quadratic systems with a weak focus, Rapport de Recherche du Département de Mathématiques et de Statistique, D.M.S. No 89-19, Univérsité de Montréal, 1989. 
35. __ Une caractérisation géométrique générique des champs de vecteurs quadratiques avec un centre, C. R. Acad. Sci. Paris Ser. I Math. 310 (1990), 723-726.

36. __ Algebraic integrals of quadratic systems with a weak focus, Proc. Conf. on Bifurcations of Planar Vector Fields, Luminy, 1989, Lecture Notes in Math., vol. 1455, Springer-Verlag, Berlin and New York, 1991, pp. 373-384.

37. D. Schlomiuk, J. Guckenheimer, and R. Rand, Integrability of plane quadratic vector fields, Exposition. Math. 8 (1990), 3-25.

38. Shi Songling, A counterexample to Chin's proposed solution to Hilbert's 16 th problem, Bull. London Math. Soc. 20 (1988), 597-599.

39. _ _ A method of constructing cycles without contact around a weak focus, J. Differential Equations 41 (1981), 301-312.

40. __ Hilbert's sixteenth problem (the second part): its present state, Ann. Sci. Math. Québec 14 (1990), 193-206.

41. M. F. Singer, Liouvillian first integrals of differential equations, Trans. Amer. Math. Soc. 333 (1992), 673-688.

42. M. Stillman, M. Stillman, and D. Bayer Macauley user manual, preprint, 1989.

43. Date Tsutomu, Classification and analysis of two-dimensional real homogeneous quadratic differential equation systems, J. Differential Equations 32 (1979), 311-334.

44. N. I. Vulpe, Affine-invariant conditions for the topological discrimination of quadratic systems with a center, transl. from Differentsial'nye Uravneniya 19 (1983), no. 3, 371-379.

45. Yan-Qian Ye et al., The theory of limit cycles, Transl. Math. Monographs, vol. 66, Amer. Math. Soc., Providence, R.I., 1984.

Département de Mathématiques et de Statistique, Univérsité de Montréal, Montréal, QUÉBEC H3C 3J7, CANADA

E-mail address:dasch@mathcn.umontreal.ca 